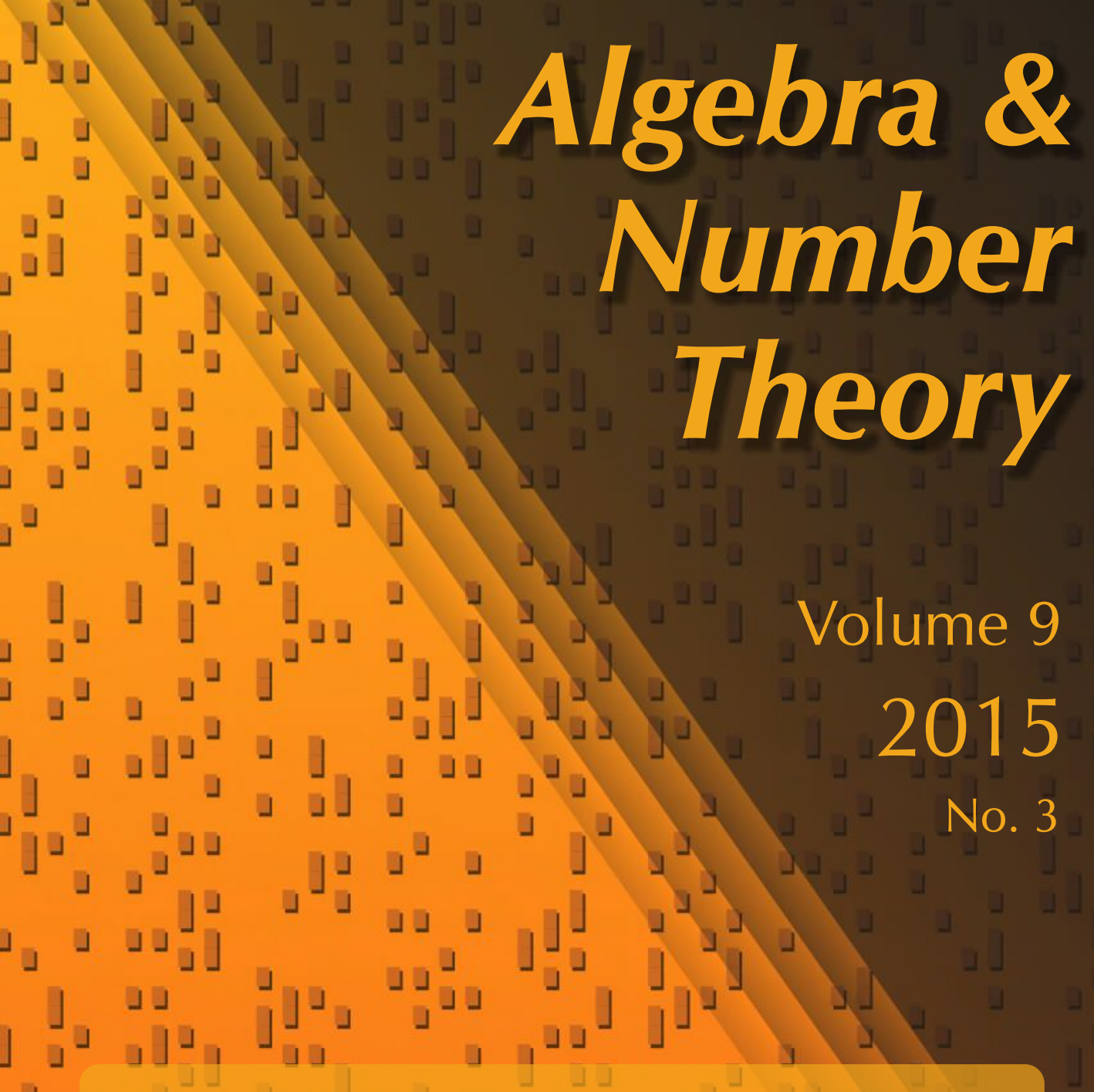

Complex group algebras of the double covers of the symmetric and alternating groups

Christine Bessenrodt, Hung Ngoc Nguyen, Jørn B. Olsson and Hung P. Tong-Viet

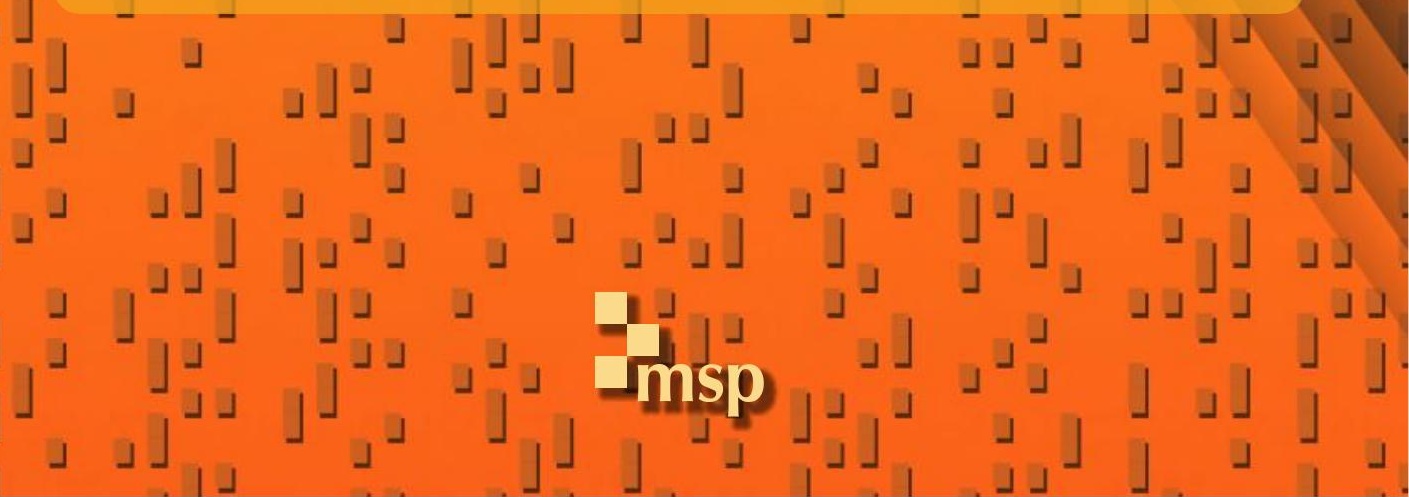




\title{
Complex group algebras of the double covers of the symmetric and alternating groups
}

\author{
Christine Bessenrodt, Hung Ngoc Nguyen, \\ Jørn B. Olsson and Hung P. Tong-Viet
}

\begin{abstract}
We prove that the double covers of the alternating and symmetric groups are determined by their complex group algebras. To be more precise, let $n \geq 5$ be an integer, $G$ a finite group, and let $\widehat{\mathrm{A}}_{n}$ and $\widehat{\mathrm{S}}_{n}^{ \pm}$denote the double covers of $\mathrm{A}_{n}$ and $\mathrm{S}_{n}$, respectively. We prove that $\mathbb{C} G \cong \mathbb{C} \widehat{\mathrm{A}}_{n}$ if and only if $G \cong \widehat{\mathrm{A}}_{n}$, and $\mathbb{C} G \cong \mathbb{C} \hat{S}_{n}^{+} \cong \mathbb{C} \widehat{S}_{n}^{-}$if and only if $G \cong \hat{\mathrm{S}}_{n}^{+}$or $\hat{\mathrm{S}}_{n}^{-}$. This in particular completes the proof of a conjecture proposed by the second and fourth authors that every finite quasisimple group is determined uniquely up to isomorphism by the structure of its complex group algebra. The known results on prime power degrees and relatively small degrees of irreducible (linear and projective) representations of the symmetric and alternating groups together with the classification of finite simple groups play an essential role in the proofs.
\end{abstract}

\section{Introduction}

The complex group algebra of a finite group $G$, denoted by $\mathbb{C} G$, is the set of formal sums $\left\{\sum_{g \in G} a_{g} g \mid a_{g} \in \mathbb{C}\right\}$, equipped with natural rules for addition, multiplication, and scalar multiplication. Wedderburn's theorem implies that $\mathbb{C} G$ is isomorphic to the direct sum of matrix algebras over $\mathbb{C}$ whose dimensions are exactly the degrees of the (nonisomorphic) irreducible complex representations of $G$. Therefore, the study of complex group algebras and the relation to their base groups is important in group representation theory.

In an attempt to understand the connection between the structure of a finite group and its complex group algebra, in Question 2 of the landmark paper [Brauer 1963]

Nguyen was partially supported by the NSA Young Investigator grant H98230-14-1-0293 and a Faculty Scholarship Award from Buchtel College of Arts and Sciences at The University of Akron. Tong-Viet's work is based on the research supported in part by the National Research Foundation of South Africa (grant 93408).

MSC2010: primary 20C30; secondary 20C15, $20 \mathrm{C} 33$.

Keywords: symmetric groups, alternating groups, complex group algebras, Schur covers, double covers, irreducible representations, character degrees. 
it was asked: when do nonisomorphic groups have isomorphic complex group algebras? Since this question might be too general to be solved completely, it is more feasible to study more explicit questions/problems whose solutions will provide a partial answer to Brauer's question. For instance, if two finite groups have isomorphic complex group algebras and one of them is solvable, is it true that the other is also solvable? Or, if two finite groups have isomorphic complex group algebras and one of them has a normal Sylow $p$-subgroup, can we conclude the same for the other group? We refer the reader to [Brauer 1963] or Section 9 of the survey paper [Navarro 2010] for more discussions on complex group algebras.

A natural problem that arises from Brauer's question is the following: given a finite group $G$, determine all finite groups (up to isomorphism) with complex group algebras isomorphic to that of $G$. This problem is easy for abelian groups but difficult for solvable groups in general. If $G$ is any finite abelian group of order $n$, then $\mathbb{C} G$ is isomorphic to a direct sum of $n$ copies of $\mathbb{C}$, so the complex group algebras of any two abelian groups are isomorphic if and only if the two groups have the same order. For solvable groups, the probability that two groups have isomorphic complex group algebra is often fairly "high". For instance, it was pointed out in [Huppert 2000] that among 2328 groups of order $2^{7}$, there are only 30 different complex group algebra structures. In contrast to solvable groups, simple groups or more generally quasisimple groups seem to have a stronger connection to their complex group algebras. In [Nguyen and Tong-Viet 2014], two of the four current authors have conjectured that every finite quasisimple group is determined uniquely up to isomorphism by its complex group algebra, and proved it for all quasisimple groups except the nontrivial perfect central covers of the alternating groups.

Let $A_{n}$ denote the alternating group of degree $n$. (Throughout the paper we always assume that $n \geq 5$, unless otherwise stated.) The Schur covers (or covering groups) of the alternating and symmetric groups were first studied and classified in [Schur 1911] in connection with their projective representations. It is known that $A_{n}$ has one isomorphism class of Schur covers, which is indeed the double cover $\widehat{A}_{n}$ except when $n$ is 6 or 7, where triple and 6 -fold covers also exist. We are able to prove that every double cover of an alternating group of degree at least 5 is determined uniquely by its complex group algebra.

Theorem A. Let $n \geq 5$. Let $G$ be a finite group and $\widehat{\mathrm{A}}_{n}$ the double cover of $\mathrm{A}_{n}$. Then $G \cong \widehat{\mathrm{A}}_{n}$ if and only if $\mathbb{C} G \cong \mathbb{C} \widehat{\mathrm{A}}_{n}$.

We prove a similar result for the triple and 6-fold (perfect central) covers of $A_{6}$ and $A_{7}$, and therefore complete the proof of the aforementioned conjecture.

Theorem B. Let $G$ be a finite group and $H$ a quasisimple group. Then $G \cong H$ if and only if $\mathbb{C} G \cong \mathbb{C} H$. 
Proof. This is a consequence of Theorem A, Theorem 6.2, and [Nguyen and TongViet 2014, Corollary 1.4].

The symmetric group $S_{n}$ has two isomorphism classes of Schur double covers, denoted by $\widehat{S}_{n}^{-}$and $\widehat{S}_{n}^{+}$. It turns out that these two covers are isoclinic, and therefore their complex group algebras $\mathbb{C} \widehat{S}_{n}^{+}$and $\mathbb{C} \widehat{S}_{n}^{-}$are isomorphic [Morris 1962]. Our next result solves the above problem for the double covers of the symmetric groups.

Theorem C. Let $n \geq 5$. Let $G$ be a finite group and $\hat{\mathrm{S}}_{n}^{ \pm}$the double covers of $\mathrm{S}_{n}$. Then $\mathbb{C} G \cong \mathbb{C} \widehat{S}_{n}^{+}$(or equivalently $\mathbb{C} G \cong \mathbb{C}_{n}^{-}$) if and only if $G \cong \widehat{\mathrm{S}}_{n}^{+}$or $G \cong \widehat{\mathrm{S}}_{n}^{-}$.

Let $\operatorname{Irr}(G)$ denote the set of all irreducible representations (or characters) of a group $G$ over the complex field. As mentioned above, two finite groups have isomorphic complex group algebras if and only if they have the same set of degrees (counting multiplicities) of irreducible characters. Therefore, the proofs of our main results as expected depend heavily on the representation theories of the symmetric and alternating groups, their double covers, and quasisimple groups in general. In particular, we make use of known results on relatively small degrees and prime power degrees of the irreducible characters of $\widehat{A}_{n}$ and $\widehat{S}_{n}^{ \pm}$.

The remainder of the paper is organized as follows. In the next section, we give a brief overview of the representation theory of the symmetric and alternating groups and their double covers, and then collect some results on prime power character degrees of these groups. The results on minimal degrees are then presented in Section 3. In Section 4, we establish some useful lemmas that will be needed later in the proofs of the main results. The proof of Theorem A is carried out in Section 5 and exceptional covers of $A_{6}$ and $A_{7}$ are treated in Section 6.

The last four sections are devoted to the proof of Theorem C. Let $G$ be a finite group such that $\mathbb{C} G \cong \mathbb{C} \widehat{S}_{n}^{ \pm}$. We will show that $G^{\prime}=G^{\prime \prime}$, and therefore there exists a normal subgroup $M$ of $G$ such that $M \subseteq G^{\prime}$ and the chief factor $G^{\prime} / M$ is isomorphic to a direct product of $k$ copies of a nonabelian simple group $S$. To prove that $G$ is isomorphic to one of $\widehat{S}_{n}^{ \pm}$, one of the key steps is to show that this chief factor is isomorphic to $A_{n}$. We will do this by using the classification of finite simple groups to eliminate almost all possibilities for $k$ and $S$. As we will see, it turns out that the case of simple groups of Lie type in even characteristic is most difficult.

In Section 7, we prove a nonexistence result for particular character degrees of $\widehat{\mathrm{S}}_{n}^{ \pm}$, and we apply it in Section 8 to show that $S$ cannot be a simple group of Lie type in even characteristic. We then eliminate other possibilities for $S$ in Section 9, and complete the proof of Theorem C in Section 10.

Notation. Since $\mathbb{C} \widehat{S}_{n}^{+} \cong \mathbb{C} \widehat{S}_{n}^{-}$, when working with character degrees of $\widehat{S}_{n}^{ \pm}$, it suffices to consider just one of the two covers. For the sake of convenience, we will write $\widehat{\mathrm{S}}_{n}$ to denote either one of the two double covers of $\mathrm{S}_{n}$. If $X$ and $Y$ are two multisets, we write $X \subseteq Y$, and say that $X$ is a submultiset of $Y$, if the multiplicity 
of any element in $X$ does not exceed that of the same element in $Y$. For a finite group $G$, the number of conjugacy classes of $G$ is denoted by $k(G)$. We write $\operatorname{Irr}_{2^{\prime}}(G)$ to mean the set of all irreducible characters of $G$ of odd degree. The set and the multiset of character degrees of $G$ are denoted respectively by $\operatorname{cd}(G)$ and $\operatorname{cd}^{*}(G)$. Finally, we denote by $d_{i}(G)$ the $i$-th smallest nontrivial character degree of $G$. Other notation is standard or will be defined when needed.

\section{Prime power degrees of $\widehat{S}_{n}$ and $\widehat{A}_{n}$}

In this section, we collect some results on irreducible characters of prime power degree of $\widehat{S}_{n}$ and $\widehat{A}_{n}$. The irreducible characters of the double covers of the symmetric and alternating groups are divided into two kinds: faithful characters, which are also known as spin characters, and nonfaithful characters, which can be viewed as ordinary characters of $S_{n}$ or $A_{n}$.

2A. Characters of $S_{n}$ and $A_{n}$. To begin with, let us recall some notation and terminology of partitions and Young diagrams in connection with representation theory of the symmetric and alternating groups. A partition $\lambda$ of $n$ is a finite sequence of natural numbers $\left(\lambda_{1}, \lambda_{2}, \ldots, \lambda_{m}\right)$ such that $\lambda_{1} \geq \lambda_{2} \geq \cdots \geq \lambda_{m}$ and $\lambda_{1}+\lambda_{2}+\cdots+\lambda_{m}=n$. If $\lambda_{1}>\lambda_{2}>\cdots>\lambda_{m}$, we say that $\lambda$ is a bar partition of $n$ (also called a strict partition of $n$ ). The Young diagram associated to $\lambda$ is an array of $n$ nodes with $\lambda_{i}$ nodes on the $i$-th row. At each node $(i, j)$, we define the hook length $h(i, j)$ to be the number of nodes to the right and below the node $(i, j)$, including the node $(i, j)$.

It is well known that the irreducible characters of $S_{n}$ are in one-to-one correspondence with partitions of $n$. The degree of the character $\chi_{\lambda}$ corresponding to $\lambda$ is given by the hook-length formula of Frame, Robinson and Thrall [Frame et al. 1954]:

$$
f_{\lambda}:=\chi_{\lambda}(1)=\frac{n !}{\prod_{i, j} h(i, j)} .
$$

Two partitions of $n$ whose Young diagrams transform into each other when reflected about the line $y=-x$, with the coordinates of the upper-left node taken to be $(0,0)$, are called conjugate partitions. The partition conjugate to $\lambda$ is denoted by $\bar{\lambda}$. If $\lambda=\bar{\lambda}$, we say that $\lambda$ is self-conjugate. The irreducible characters of $\mathrm{A}_{n}$ can be obtained by restricting those of $S_{n}$ to $A_{n}$. More explicitly, $\chi_{\lambda} \downarrow_{A_{n}}=\chi_{\bar{\lambda}} \downarrow_{A_{n}}$ is irreducible if $\lambda$ is not self-conjugate. Otherwise, $\chi_{\lambda} \downarrow_{A_{n}}$ is the sum of two different irreducible characters of $A_{n}$ of the same degree. In short, the degrees of irreducible characters of $\mathrm{A}_{n}$ are labeled by partitions of $n$ and are given by

$$
\tilde{f}_{\lambda}= \begin{cases}f_{\lambda} & \text { if } \lambda \neq \bar{\lambda} \\ f_{\lambda} / 2 & \text { if } \lambda=\bar{\lambda}\end{cases}
$$


Irreducible representations of prime power degree of the symmetric and alternating groups were classified by A. Balog, C. Bessenrodt, J. B. Olsson and K. Ono [Balog et al. 2001]. This result is critical in eliminating simple groups other than $\mathrm{A}_{n}$ involved in the structure of finite groups whose complex group algebras are isomorphic to $\mathbb{C} \widehat{A}_{n}$ or $\mathbb{C} \widehat{S}_{n}$.

Lemma 2.1 [Balog et al. 2001, Theorem 2.4]. Let $n \geq 5$. An irreducible character $\chi_{\lambda} \in \operatorname{Irr}\left(\mathrm{S}_{n}\right)$ corresponding to a partition $\lambda$ of $n$ has prime power degree $f_{\lambda}=p^{r}>1$ if and only if one of the following occurs:

(1) $n=p^{r}+1, \lambda=(n-1,1)$ or $\left(2,1^{n-2}\right)$, and $f_{\lambda}=n-1$.

(2) $n=5, \lambda=\left(2^{2}, 1\right)$ or $(3,2)$, and $f_{\lambda}=5$.

(3) $n=6, \lambda=(4,2)$ or $\left(2^{2}, 1^{2}\right)$, and $f_{\lambda}=9 ; \lambda=\left(3^{2}\right)$ or $\left(2^{3}\right)$, and $f_{\lambda}=5$; $\lambda=(3,2,1)$ and $f_{\lambda}=16$.

(4) $n=8, \lambda=(5,2,1)$ or $\left(3,2,1^{3}\right)$, and $f_{\lambda}=64$.

(5) $n=9, \lambda=(7,2)$ or $\left(2^{2}, 1^{5}\right)$, and $f_{\lambda}=27$.

Lemma 2.2 [Balog et al. 2001, Theorem 5.1]. Let $n \geq 5$. An irreducible character degree $\tilde{f}_{\lambda}$ of $\mathrm{A}_{n}$ corresponding to a partition $\lambda$ of $n$ is a prime power $p^{r}>1$ if and only if one of the following occurs:

(1) $n=p^{r}+1, \lambda=(n-1,1)$ or $\left(2,1^{n-2}\right)$, and $\tilde{f}_{\lambda}=n-1$.

(2) $n=5, \lambda=\left(2^{2}, 1\right)$ or $(3,2)$, and $\tilde{f}_{\lambda}=5 ; \lambda=\left(3,1^{2}\right)$ and $\tilde{f}_{\lambda}=3$.

(3) $n=6, \lambda=(4,2)$ or $\left(2^{2}, 1^{2}\right)$ and $\tilde{f}_{\lambda}=9 ; \lambda=\left(3^{2}\right)$ or $\left(2^{3}\right)$ and $\tilde{f}_{\lambda}=5$; $\lambda=(3,2,1)$ and $\tilde{f}_{\lambda}=8$.

(4) $n=8, \lambda=(5,2,1)$ or $\left(3,2,1^{3}\right)$, and $\tilde{f}_{\lambda}=64$.

(5) $n=9, \lambda=(7,2)$ or $\left(2^{2}, 1^{5}\right)$, and $\tilde{f}_{\lambda}=27$.

2B. Spin characters of $S_{\boldsymbol{n}}$ and $\mathrm{A}_{\boldsymbol{n}}$. We now recall the spin representation theory of the symmetric and alternating groups, due to Schur [Hoffman and Humphreys 1992; Morris 1962; Schur 1911; Wagner 1977]. To each bar partition $\mu=$ $\left(\mu_{1}, \mu_{2}, \ldots, \mu_{m}\right)$ (i.e., $\left.\mu_{1}>\mu_{2}>\cdots>\mu_{m}\right)$ of $n$, there corresponds one or two irreducible characters (depending on whether $n-m$ is even or odd, respectively) of $\widehat{S}_{n}$, of degree

$$
g_{\mu}=2^{\lfloor(n-m) / 2\rfloor} \bar{g}_{\mu}
$$

where $\bar{g}_{\mu}$ denotes the number of shifted standard tableaux of shape $\mu$. This number can be computed by an analogue of the hook-length formula, the bar formula [Hoffman and Humphreys 1992, Proposition 10.6]. The length $b(i, j)$ of the $(i, j)$ bar of $\mu$ is the length of the $(i, j+1)$-hook in the shift-symmetric diagram of $\mu$ 
(obtained by reflecting the shifted diagram of $\mu$ along the diagonal and pasting it onto $\mu$; see [Macdonald 1995, p. 14] for details). Then

$$
\bar{g}_{\mu}=\frac{n !}{\prod_{i, j} b(i, j)} .
$$

The spin character degree may also be computed by the formula

$$
g_{\mu}=2^{\lfloor(n-m) / 2\rfloor} \frac{n !}{\mu_{1} ! \mu_{2} ! \cdots \mu_{m} !} \prod_{i<j} \frac{\mu_{i}-\mu_{j}}{\mu_{i}+\mu_{j}} .
$$

Again, one can get faithful irreducible characters of $\widehat{A}_{n}$ by restricting those of $\widehat{\mathrm{S}}_{n}^{ \pm}$to $\widehat{\mathrm{A}}_{n}$ in the following way. If $n-m$ is odd, then the restrictions of the two characters of $\widehat{S}_{n}^{ \pm}$labeled by $\mu$ to $\widehat{A}_{n}$ are the same and irreducible. Otherwise, the restriction of the one character labeled by $\mu$ is the sum of two irreducible characters of the same degree $g_{\mu} / 2$. Let $\tilde{g}_{\mu}$ be the degree of the irreducible spin character(s) of $\widehat{\mathrm{A}}_{n}$ labeled by the bar partition $\mu$; we then have

$$
\tilde{g}_{\mu}= \begin{cases}g_{\mu} & \text { if } n-m \text { is odd, } \\ g_{\mu} / 2 & \text { if } n-m \text { is even. }\end{cases}
$$

The classification of spin representations of prime power degree of the symmetric and alternating groups has been done by the first and third authors of the current paper in [Bessenrodt and Olsson 2002].

Lemma 2.3 [Bessenrodt and Olsson 2002, Theorem 4.2]. Let $n \geq 5$, and let $\mu$ be a bar partition of $n$. The spin irreducible character degree $g_{\mu}$ of $\widehat{\mathrm{S}}_{n}$ corresponding to $\mu$ is a prime power if and only if one of the following occurs:

(1) $\mu=(n)$ and $g_{\mu}=2^{\lfloor(n-1) / 2\rfloor}$.

(2) $n=2^{r}+2$ for some $r \in \mathbb{N}, \mu=(n-1,1)$, and $g_{\mu}=2^{2^{r-1}+r}$.

(3) $n=5, \mu=(3,2)$, and $g_{\mu}=4$.

(4) $n=6, \mu=(3,2,1)$, and $g_{\mu}=4$.

(5) $n=8, \mu=(5,2,1)$, and $g_{\mu}=64$.

Lemma 2.4 [Bessenrodt and Olsson 2002, Theorem 4.3]. Let $n \geq 5$, and let $\mu$ be a bar partition of $n$. The spin irreducible character degree $\tilde{g}_{\mu}$ of $\widehat{\mathrm{A}}_{n}$ corresponding to $\mu$ is a prime power if and only if one of the following occurs:

(1) $\mu=(n)$ and $\tilde{g}_{\mu}=2^{\lfloor(n-2) / 2\rfloor}$.

(2) $n=2^{r}+2$ for some $r \in \mathbb{N}, \mu=(n-1,1)$, and $\tilde{g}_{\mu}=2^{2^{r-1}+r-1}$.

(3) $n=5, \mu=(3,2)$, and $\tilde{g}_{\mu}=4$.

(4) $n=6, \mu=(3,2,1)$, and $\tilde{g}_{\mu}=4$.

(5) $n=8, \mu=(5,2,1)$, and $\tilde{g}_{\mu}=64$. 


\section{Low degrees of $\widehat{S}_{n}$ and $\widehat{A}_{n}$}

We present in this section some results on minimal degrees of both ordinary and spin characters of the symmetric and alternating groups. We start with ordinary characters:

Lemma 3.1 [Rasala 1977]. The following hold:

(1) $d_{1}\left(\mathrm{~S}_{n}\right)=n-1$ if $n \geq 5$.

(2) $d_{2}\left(\mathrm{~S}_{n}\right)=n(n-3) / 2$ if $n \geq 9$.

(3) $d_{3}\left(\mathrm{~S}_{n}\right)=(n-1)(n-2) / 2$ if $n \geq 9$.

(4) $d_{4}\left(\mathrm{~S}_{n}\right)=n(n-1)(n-5) / 6$ if $n \geq 13$.

(5) $d_{5}\left(\mathrm{~S}_{n}\right)=(n-1)(n-2)(n-3) / 6$ if $n \geq 13$.

(6) $d_{6}\left(\mathrm{~S}_{n}\right)=n(n-2)(n-4) / 3$ if $n \geq 15$.

(7) $d_{7}\left(\mathrm{~S}_{n}\right)=n(n-1)(n-2)(n-7) / 24$ if $n \geq 15$.

Lemma 3.2 [Tong-Viet 2011]. If $n \geq 15$, then $d_{i}\left(\mathrm{~A}_{n}\right)=d_{i}\left(\mathrm{~S}_{n}\right)$ for $1 \leq i \leq 4$, and, if $n \geq 22$, then $d_{i}\left(\mathrm{~A}_{n}\right)=d_{i}\left(\mathrm{~S}_{n}\right)$ for $1 \leq i \leq 7$.

The minimal degrees of spin irreducible representations of $\widehat{A}_{n}$ and $\widehat{S}_{n}$ were obtained in [Kleshchev and Tiep 2004; 2012]. These minimal degrees are indeed the degrees of the basic spin and second basic spin representations. Let $\mathfrak{d}_{1}\left(\widehat{\mathrm{A}}_{n}\right)$ and $\mathfrak{d}_{1}\left(\widehat{\mathrm{S}}_{n}\right)$ denote the smallest degrees of irreducible spin characters of $\widehat{A}_{n}$ and $\widehat{S}_{n}$, respectively.

Lemma 3.3 [Kleshchev and Tiep 2004, Theorem A]. Let $n \geq 8$. The smallest degrees of the irreducible spin characters of $\widehat{\mathrm{A}}_{n}$ and $\widehat{\mathrm{S}}_{n}$ are $\mathfrak{d}_{1}\left(\widehat{\mathrm{A}}_{n}\right)=2^{\lfloor(n-2) / 2\rfloor}$ and $\mathfrak{d}_{1}\left(\widehat{\mathrm{S}}_{n}\right)=2^{\lfloor(n-1) / 2\rfloor}$ respectively, and there is no degree between $\mathfrak{d}_{1}\left(\widehat{\mathrm{A}}_{n}\right)$ and $2 \mathfrak{d}_{1}\left(\widehat{\mathrm{A}}_{n}\right)$ in the alternating case or $\mathfrak{d}_{1}\left(\widehat{\mathrm{S}}_{n}\right)$ and $2 \mathfrak{d}_{1}\left(\widehat{\mathrm{S}}_{n}\right)$ in the symmetric one.

Using the above results, we easily deduce the following:

Lemma 3.4. The following hold:

(1) If $n \geq 31$, then $d_{i}\left(\widehat{\mathrm{S}}_{n}\right)=d_{i}\left(\mathrm{~S}_{n}\right)$ for $1 \leq i \leq 7$.

(2) If $n \geq 34$, then $d_{i}\left(\widehat{\mathrm{A}}_{n}\right)=d_{i}\left(\mathrm{~A}_{n}\right)$ for $1 \leq i \leq 7$.

Proof. We observe that $\mathfrak{d}_{1}\left(\widehat{\mathrm{S}}_{n}\right)=2^{\lfloor(n-1) / 2\rfloor}>n(n-1)(n-2)(n-7) / 24=d_{7}\left(\mathrm{~S}_{n}\right)$ if $n \geq 31$. Therefore part (1) follows by Lemmas 3.1 and 3.3. Similarly, we have $\mathfrak{d}_{1}\left(\widehat{\mathrm{A}}_{n}\right)=2^{\lfloor(n-2) / 2\rfloor}>n(n-1)(n-2)(n-7) / 24=d_{7}\left(\mathrm{~A}_{n}\right)$ if $n \geq 34$, and thus part (2) follows.

Lemma 3.5. Let $G$ be either $\widehat{\mathrm{A}}_{n}$ or $\widehat{\mathrm{S}}_{n}$.

(1) If $n \geq 8$, then $d_{1}(G)=n-1$.

(2) If $n \geq 10$, then $d_{2}(G)=\min \left\{n(n-3) / 2, \mathfrak{d}_{1}(G)\right\}$. Furthermore, if $n \geq 12$, then $d_{2}(G)>2 n$. 
(3) If $n \geq 16$, then $d_{3}(G)=(n-1)(n-2) / 2$ and $d_{4}(G)=\min \{n(n-1)(n-5) / 6$, $\left.\mathfrak{d}_{1}(G)\right\}$.

(4) If $n \geq 28$, then $d_{4}(G)=n(n-1)(n-5) / 6, d_{5}(G)=(n-1)(n-2)(n-3) / 6$, $d_{6}(G)=n(n-2)(n-4) / 3$, and $d_{7}(G)=\min \left\{n(n-1)(n-2)(n-7) / 24, \mathfrak{d}_{1}(G)\right\}$.

Proof. When $n \geq 8$, we see that $\mathfrak{d}_{1}\left(\widehat{\mathrm{S}}_{n}\right) \geq \mathfrak{d}_{1}\left(\widehat{\mathrm{A}}_{n}\right)=2^{\lfloor(n-2) / 2\rfloor}>n-1$ and $d_{1}\left(\mathrm{~S}_{n}\right)=d_{1}\left(\mathrm{~A}_{n}\right)=n-1$, which implies that $d_{1}\left(\widehat{\mathrm{A}}_{n}\right)=d_{1}\left(\widehat{\mathrm{S}}_{n}\right)=n-1$, and part $(1)$ follows. When $n \geq 10$, we observe that $d_{2}\left(\mathrm{~A}_{n}\right)=d_{2}\left(\mathrm{~S}_{n}\right)=n(n-3) / 2$, and part (2) then follows from part (1).

Suppose that $n \geq 16$. It is easy to check that $2^{\lfloor(n-2) / 2\rfloor}>(n-1)(n-2) / 2=$ $d_{3}\left(\mathrm{~A}_{n}\right)=d_{3}\left(\mathrm{~S}_{n}\right)$. It follows that $d_{3}\left(\widehat{\mathrm{A}}_{n}\right)=d_{3}\left(\widehat{\mathrm{S}}_{n}\right)=(n-1)(n-2) / 2$ and $d_{4}(G)=$ $\min \left\{n(n-1)(n-5) / 6, \mathfrak{d}_{1}(G)\right\}$, as claimed.

Finally suppose that $n \geq 28$. We check that $2^{\lfloor(n-2) / 2\rfloor}>n(n-2)(n-4) / 3=$ $d_{6}\left(\mathrm{~A}_{n}\right)=d_{6}\left(\mathrm{~S}_{n}\right)$, and part (4) then follows.

\section{Some useful lemmas}

We begin with an easy observation:

Lemma 4.1. There always exists a prime number $p$ with $n<p \leq d_{2}(G)$ for $G=\widehat{\mathrm{A}}_{n}$ or $\widehat{\mathrm{S}}_{n}$, provided that $n \geq 9$.

Proof. It is routine to check the statement for $9 \leq n \leq 11$ by using [Conway et al. 1985]. So we can assume that $n \geq 12$. By Lemma 3.5, it suffices to prove that there exists a prime between $n$ and $2 n$. However, this is the well-known BertrandChebyshev theorem [Harborth and Kemnitz 1981].

The next three lemmas are critical in the proof of Theorem A.

Lemma 4.2. Let $S$ be a simple group of Lie type of rank $l$ defined over a field of $q$ elements, with q even. Then

$$
\left|\operatorname{Irr}_{2^{\prime}}(S)\right| \geq \begin{cases}q^{l} /(l+1, q-1) & \text { if } S \text { is of type } A, \\ q^{l} /(l+1, q+1) & \text { if } S \text { is of type }{ }^{2} A, \\ q^{l} / 3 & \text { if } S \text { is of type } E_{6},{ }^{2} E_{6}, \\ q^{l} & \text { otherwise. }\end{cases}
$$

Proof. Let $S_{\text {sc }}$ be the finite Lie-type group of simply connected type corresponding to $S$. By [Brunat 2010, Corollary 3.6], $S_{\mathrm{sc}}$ has $q^{l}$ semisimple conjugacy classes. To each semisimple class $s$ of $S_{\mathrm{sc}}$, Lusztig's classification of complex characters of finite groups of Lie type says that there corresponds a semisimple character of the dual group, say $S_{\mathrm{sc}}^{*}$, of $S_{\mathrm{sc}}$ of degree

$$
\left|S_{\mathrm{sc}}\right|_{2^{\prime}} /\left|\boldsymbol{C}_{S_{\mathrm{sc}}}(s)\right|_{2^{\prime}}
$$


This means that the dual $S_{\mathrm{sc}}^{*}$ of $S_{\mathrm{sc}}$ has at least $q^{l}$ irreducible characters of odd degree.

If $S$ is of type $A$, we have $S_{\mathrm{sc}}^{*}=\operatorname{PGL}_{l+1}(q)=S .(l+1, q-1)$, and the lemma follows for linear groups. A similar argument works for unitary groups and $E_{6}$ as well as ${ }^{2} E_{6}$. If $S$ is not of these types, we will have $S=S_{\mathrm{sc}}=S_{\mathrm{sc}}^{*}$ and the lemma also follows.

Lemma 4.3. Let $n \geq 5$, and let $n=2^{k_{1}}+2^{k_{2}}+\cdots+2^{k_{t}}$ be the binary expansion of $n$, with $k_{1}>k_{2}>\cdots>k_{t} \geq 0$. Then

$$
\left|\operatorname{Irr}_{2^{\prime}}\left(\widehat{\mathrm{A}}_{n}\right)\right| \leq\left|\operatorname{Irr}_{2^{\prime}}\left(\widehat{\mathrm{S}}_{n}\right)\right|=2^{k_{1}+k_{2}+\cdots+k_{t}} .
$$

Proof. If $\mu=\left(\mu_{1}, \mu_{2}, \ldots, \mu_{m}\right)$ is a bar partition of $n$, then the 2-part of the spin character degree $g_{\mu}$ of $\widehat{S}_{n}$ labeled by $\mu$ is at least

$$
2^{\lfloor(n-m) / 2\rfloor},
$$

which is at least 2 as $n \geq 5$ and $n-m \geq 3$. We note that if $n-m=3$ then $\tilde{g}_{\mu}=g_{\mu}$. Therefore, the 2-part of the degree $\tilde{g}_{\mu}$ of $\widehat{A}_{n}$ is at least 2 as well. In particular, we see that every spin character degree of $\widehat{\mathrm{A}}_{n}$ as well as $\widehat{\mathrm{S}}_{n}$ is even. It follows that

$$
\left|\operatorname{Irr}_{2^{\prime}}\left(\widehat{\mathrm{A}}_{n}\right)\right|=\left|\operatorname{Irr}_{2^{\prime}}\left(\mathrm{A}_{n}\right)\right| \text { and }\left|\operatorname{Irr}_{2^{\prime}}\left(\widehat{\mathrm{S}}_{n}\right)\right|=\left|\operatorname{Irr}_{2^{\prime}}\left(\mathrm{S}_{n}\right)\right| .
$$

As mentioned in [McKay 1972], the number of odd degree irreducible characters of $A_{n}$ does not exceed that of $S_{n}$. Now the lemma follows from the formula for the number of odd degree characters of $S_{n}$ given in [Macdonald 1971, Corollary 1.3].

Lemma 4.4. Let $n=2^{k_{1}}+2^{k_{2}}+\cdots+2^{k_{t}}$ be the binary expansion of $n$, with $k_{1}>k_{2}>\cdots>k_{t} \geq 0$.

(1) If $k_{1}+k_{2}+\cdots+k_{t} \geq \sqrt{(n-3) / 2}$, then $n<2^{15}$.

(2) if $k_{1}+k_{2}+\cdots+k_{t} \geq \sqrt{n-3}-3$, then $n<2^{13}$.

(3) if $k_{1}+k_{2}+\cdots+k_{t} \geq(n-3) / 18$, then $n<2^{10}$.

(4) if $k_{1}+k_{2}+\cdots+k_{t} \geq(n-3) / 30$, then $n<2^{11}$.

Proof. We only give here a proof for part (2). The other statements are proved similarly. As $n=2^{k_{1}}+2^{k_{2}}+\cdots+2^{k_{t}}$, we get $k_{1}=\left\lfloor\log _{2} n\right\rfloor$ and hence

$$
k_{1}+k_{2}+\cdots+k_{t} \geq\left\lfloor\log _{2} n\right\rfloor\left(\left\lfloor\log _{2} n\right\rfloor+1\right) / 2 .
$$

However, it is easy to check that $\sqrt{(n-3)}-3>\left\lfloor\log _{2} n\right\rfloor\left(\left\lfloor\log _{2} n\right\rfloor+1\right) / 2$ if $n \geq 2^{14}$. For $2^{13} \leq n<2^{14}$, the statement follows by direct computation.

The following lemma is probably known, but we include a short proof for the reader's convenience. It will be needed in the proof of Theorem C. 
Lemma 4.5. Let $k\left(\widehat{\mathrm{A}}_{n}\right)$ and $k\left(\widehat{\mathrm{S}}_{n}\right)$ denote the number of conjugacy classes of $\widehat{\mathrm{A}}_{n}$ and $\widehat{\mathrm{S}}_{n}$, respectively. Then

$$
k\left(\widehat{\mathrm{S}}_{n}\right)<2 k\left(\widehat{\mathrm{A}}_{n}\right) .
$$

Proof. Let $\operatorname{Irr}_{\text {faithful }}(G)$ and $\operatorname{Irr}_{\text {nonfaithful }}(G)$ denote the sets of faithful irreducible characters and nonfaithful irreducible characters, respectively, of a group $G$. Let $a$ and $b$ be the numbers of self-conjugate partitions and of pairs of nonself-conjugate partitions, respectively, of $n$. Also, let $c$ and $d$ be the numbers of bar partitions of $n$ with $n-m$ even and odd, respectively. We have

$$
\begin{array}{ll}
\left|\operatorname{Irr}_{\text {nonfaithful }}\left(\widehat{\mathrm{S}}_{n}\right)\right|=k\left(\mathrm{~S}_{n}\right)=a+2 b, & \left|\operatorname{Irr}_{\text {faithful }}\left(\widehat{\mathrm{S}}_{n}\right)\right|=c+2 d, \\
\left|\operatorname{Irr}_{\text {nonfaithful }}\left(\widehat{\mathrm{A}}_{n}\right)\right|=k\left(\mathrm{~A}_{n}\right)=2 a+b, & \left|\operatorname{Irr}_{\text {faithful }}\left(\widehat{\mathrm{A}}_{n}\right)\right|=2 c+d .
\end{array}
$$

Therefore,

$$
k\left(\widehat{\mathrm{S}}_{n}\right)=a+2 b+c+2 d \quad \text { and } \quad k\left(\widehat{\mathrm{A}}_{n}\right)=2 a+b+2 c+d,
$$

and the lemma follows.

\section{Complex group algebra of $\widehat{A}_{n}-$ Theorem $A$}

The aim of this section is to prove Theorem A. Let $G$ be a finite group such that $\mathbb{C} G \cong \mathbb{C} \widehat{A}_{n}$. Then $G$ has exactly one linear character, which is the trivial one, so that $G$ is perfect. Let $M$ be a maximal normal subgroup of $G$. We then have that $G / M$ is nonabelian simple, and moreover

$$
\operatorname{cd}^{*}(G / M) \subseteq \operatorname{cd}^{*}(G)=\operatorname{cd}^{*}\left(\widehat{\mathrm{A}}_{n}\right) .
$$

To prove the theorem, it is clear that we first have to show $G / M \cong \mathrm{A}_{n}$. We will work towards this aim.

Proposition 5.1. Let $S$ be a nonabelian simple group such that $\operatorname{cd}^{*}(S) \subseteq \operatorname{cd}^{*}\left(\widehat{\mathrm{A}}_{n}\right)$. Then $S$ is isomorphic to $\mathrm{A}_{n}$ or to a simple group of Lie type in even characteristic.

Proof. We will eliminate other possibilities for $S$ by using the classification of finite simple groups. If $5 \leq n \leq 9$, then the set of prime divisors of $S$ is contained in that of $\widehat{A}_{n}$, which in turn is contained in $\{2,3,5,7\}$; hence by using [Huppert and Lempken 2000, Theorem III] and [Conway et al. 1985], the result follows easily. From now on we assume that $n \geq 10$.

(i) Alternating groups: Suppose that $S=\mathrm{A}_{m}$ with $5 \leq m \neq n$. Since $\operatorname{cd}\left(A_{m}\right) \subseteq$ $\operatorname{cd}\left(\widehat{\mathrm{A}}_{n}\right)$, we get $d_{1}\left(\mathrm{~A}_{m}\right) \geq d_{1}\left(\widehat{\mathrm{A}}_{n}\right)$. As $d_{1}\left(\widehat{\mathrm{A}}_{n}\right)=n-1 \geq 9$ by Lemma 3.5 , it follows that $d_{1}\left(\mathrm{~A}_{m}\right) \geq 9$. Thus $m \geq 10$, and so $m-1=d_{1}\left(\mathrm{~A}_{m}\right) \geq d_{1}\left(\widehat{\mathrm{A}}_{n}\right)=n-1$. In particular, we have $m>n$ as $m \neq n$. It follows that $|S|>2\left|\mathrm{~A}_{n}\right|$ and this violates the hypothesis that $\operatorname{cd}^{*}(S) \subseteq \operatorname{cd}^{*}\left(\widehat{A}_{n}\right)$. 
(ii) Simple groups of Lie type in odd characteristic: Suppose that $S=G\left(p^{k}\right)$, a simple group of Lie type defined over a field of $p^{k}$ elements with $p$ odd. Since $|S|_{p}$ is the degree of the Steinberg character of $S$, we have $|S|_{p} \in \operatorname{cd}\left(\widehat{\mathrm{A}}_{n}\right)$. As $|S|_{p}$ is an odd prime power, Lemma 2.4 implies that $|S|_{p}$ must be the degree of a nonfaithful character of $\widehat{A}_{n}$. In other words, $|S|_{p} \in \operatorname{cd}\left(\mathrm{A}_{n}\right)$. Using Lemma 2.2, we deduce that $|S|_{p}=n-1$. Hence, $|S|_{p}=d_{1}\left(\widehat{\mathrm{A}}_{n}\right)$ is the smallest nontrivial degree of $\widehat{A}_{n}$ by Lemma 3.5. However, by [Tong-Viet 2012, Lemma 8] we have $d_{1}(S)<|S|_{p}=d_{1}\left(\widehat{\mathrm{A}}_{n}\right)$, which is impossible as $\operatorname{cd}(S) \subseteq \operatorname{cd}\left(\widehat{\mathrm{A}}_{n}\right)$.

(iii) Sporadic simple groups and the Tits group: Using GAP (version 4.4.12), we can assume that $n \geq 14$. To eliminate these cases, observe that $n \geq \max \{p(S), 14\}$, where $p(S)$ is the largest prime divisor of $|S|$, and that $d_{i}(S) \geq d_{i}\left(\widehat{\mathrm{A}}_{n}\right)$ for all $i \geq 1$. With this lower bound on $n$, we find the lower bounds for $d_{i}\left(\widehat{\mathrm{A}}_{n}\right)$ with $1 \leq i \leq 7$ using Lemmas 3.4 and 3.5. Choose $i \in\{2,3, \ldots, 7\}$ such that $d_{i}\left(\widehat{\mathrm{A}}_{n}\right)>d_{j}(S)$ for some $j \geq 1$ such that $|i-j|$ is minimal. If $j \geq i$, then we obtain a contradiction. If $j<i$, then $d_{j}(S) \in\left\{d_{k}\left(\widehat{\mathrm{A}}_{n}\right)\right\}_{k=j}^{i-1}$. Solving these equations for $n$, we then obtain that either these equations have no solution, or that, for each solution of $n$, we can find some $k \geq 1$ with $d_{k}\left(\widehat{\mathrm{A}}_{n}\right)>d_{k}(S)$. As an example, assume that $S=\mathrm{O}^{\prime} \mathrm{N}$. Then $n \geq 31$ since $p(S)=31$. We have $d_{7}\left(\widehat{\mathrm{A}}_{n}\right)=n(n-1)(n-2)(n-7) / 24 \geq 26970$. As $d_{4}(S)=26752<d_{7}\left(\widehat{\mathrm{A}}_{n}\right)$, it follows that $d_{4}(S) \in\left\{d_{4}\left(\widehat{\mathrm{A}}_{n}\right), d_{5}\left(\widehat{\mathrm{A}}_{n}\right), d_{6}\left(\widehat{\mathrm{A}}_{n}\right)\right\}$. However, one can check that these equations have no integer solutions.

Proposition 5.2. Let $S$ be a nonabelian simple group such that $|S| \mid n !$ and $\operatorname{cd}^{*}(S) \subseteq$ $\operatorname{cd}^{*}\left(\widehat{\mathrm{A}}_{n}\right)$. Then $S \cong \mathrm{A}_{n}$.

Proof. In light of Proposition 5.1 and its proof, it remains to assume that $n \geq 9$ and prove that $S$ cannot be a simple group of Lie type in even characteristic. Assume to the contrary that $S=G_{l}\left(2^{k}\right)$, a simple group of Lie type of rank $l$ defined over a field of $q=2^{k}$ elements. As above, we then have $|S|_{2} \in \operatorname{cd}\left(\widehat{\mathrm{A}}_{n}\right)$. By Lemmas 2.2 and 2.4, we have that $|S|_{2}=n-1,|S|_{2}=2^{\lfloor(n-2) / 2\rfloor}$, or $|S|_{2}=2^{n / 2+\log _{2}(n-2)-2}$ when $n=2^{r}+2$. Since the case $|S|_{2}=n-1$ can be eliminated as in the proof of the previous proposition, we can assume further that

$$
|S|_{2}=2^{\lfloor(n-2) / 2\rfloor} \quad \text { or } \quad|S|_{2}=2^{n / 2+\log _{2}(n-2)-2} \quad \text { when } n=2^{r}+2 .
$$

Recalling the hypothesis that $\operatorname{cd}^{*}(S) \subseteq \operatorname{cd}^{*}\left(\widehat{\mathrm{A}}_{n}\right)$, we have

$$
\left|\operatorname{Irr}_{2^{\prime}}(S)\right| \leq\left|\operatorname{Irr}_{2^{\prime}}\left(\widehat{\mathrm{A}}_{n}\right)\right| \text {. }
$$

(i) $S=B_{l}\left(2^{k}\right) \cong C_{l}\left(2^{k}\right), D_{l}\left(2^{k}\right)$, or ${ }^{2} D_{l}\left(2^{k}\right)$. Then $|S|_{2}=2^{k l^{2}}$ or $2^{k l(l-1)}$. In particular,

$$
|S|_{2} \leq 2^{k l^{2}}
$$


As $|S|_{2} \geq 2^{\lfloor(n-2) / 2\rfloor}$, it follows that

$$
k l^{2} \geq\lfloor(n-2) / 2\rfloor \geq(n-3) / 2 .
$$

Therefore

$$
k l \geq \sqrt{(n-3) / 2}
$$

Using Lemma 4.2, we then obtain

$$
\left|\operatorname{Irr}_{2^{\prime}}(S)\right| \geq q^{l}=2^{k l} \geq 2^{\sqrt{(n-3) / 2}} .
$$

Now Lemma 4.3 and the inequality (5-1) imply

$$
k_{1}+k_{2}+\cdots+k_{t} \geq \sqrt{(n-3) / 2},
$$

where $n=2^{k_{1}}+2^{k_{2}}+\cdots+2^{k_{t}}$ is the binary expansion of $n$. Invoking Lemma 4.4(1), we obtain that $n<2^{15}$.

(ii) $S=A_{l}\left(2^{k}\right)$ or ${ }^{2} A_{l}\left(2^{k}\right)$. Arguing as above, we have

$$
k_{1}+k_{2}+\cdots+k_{t} \geq \sqrt{n-3}-3,
$$

which forces $n<2^{13}$ by Lemma 4.4(2).

(iii) $S$ is a simple group of exceptional Lie type. Using Lemma 4.4(3,4), we deduce that $n<2^{11}$.

For each of the above cases, a computer program has checked that either $|S|$ does not divide $n$ ! or $S$ has an irreducible character degree not belonging to $\operatorname{cd}\left(\widehat{\mathrm{A}}_{n}\right)$ for "small" $n$. This contradiction completes the proof. Let us describe the example where $S=\operatorname{Sp}_{2 l}\left(2^{k}\right) \cong \Omega_{2 l+1}\left(2^{k}\right)$. Then we have

$$
k l^{2}=\lfloor(n-2) / 2\rfloor \quad \text { or } \quad k l^{2}=n / 2+\log _{2}(n-2)-2 \quad \text { when } n=2^{r}+2 .
$$

Moreover, the condition $|S| \mid n$ ! is equivalent to

$$
2^{k l^{2}} \prod_{i=1}^{l}\left(2^{2 k i}-1\right) \mid n ! .
$$

By computer calculations, we can determine all triples $(k, l, n)$ with $n<2^{15}$ satisfying the above conditions. It turns out that, for each such triple, $n$ is at most 170 and one of the three smallest character degrees of $S$ is not a character degree of $\widehat{A}_{n}$. The low-degree characters of simple groups of Lie type can be found in [Tiep and Zalesskii 1996; Lübeck 2001; Nguyen 2010].

We are now ready to prove the first main result: 
Proof of Theorem A. Recall the hypothesis that $G$ is a finite group such that $\mathbb{C} G \cong$ $\mathbb{C} \widehat{A}_{n}$. Therefore $\operatorname{cd}^{*}(G)=\operatorname{cd} *\left(\widehat{\mathrm{A}}_{n}\right)$. In particular, we have $|G|=\left|\widehat{\mathrm{A}}_{n}\right|$ and $G=G^{\prime}$ since $\widehat{A}_{n}$ has only one linear character. Let $M$ be a maximal normal subgroup of $G$. Then $G / M$ is a nonabelian simple group, say $S$. It follows that $\operatorname{cd}^{*}(S)=$ $\operatorname{cd}^{*}(G / M) \subseteq \operatorname{cd}^{*}(G)$ and hence

$$
\operatorname{cd}^{*}(S) \subseteq \operatorname{cd}^{*}\left(\widehat{\mathrm{A}}_{n}\right)
$$

We also have

$$
|S||| G|=| \widehat{\mathrm{A}}_{n} \mid=n ! .
$$

Applying Propositions 5.1 and 5.2, we deduce that $S \cong \mathrm{A}_{n}$.

We have shown that $G / M \cong \mathrm{A}_{n}$. Since $|G|=\left|\widehat{\mathrm{A}}_{n}\right|=2\left|\mathrm{~A}_{n}\right|$, we obtain $|M|=2$. In particular, $M$ is central in $G$ and therefore $M \subseteq Z(G) \cap G^{\prime}$. Thus $G \cong \widehat{\mathrm{A}}_{n}$, as desired.

\section{Triple and 6-fold covers of $A_{6}$ and $A_{7}$}

In this section, we aim to prove that every perfect central cover of $A_{6}$ or $A_{7}$ is uniquely determined up to isomorphism by the structure of its complex group algebra. To do that, we need the following result from [Nguyen and Tong-Viet 2014, Lemma 2.5]. Here and in what follows, we write $\operatorname{Mult}(S)$ and $\operatorname{Schur}(S)$ to denote the Schur multiplier and the Schur covering group (or the Schur cover for short), respectively, of a simple group $S$.

Lemma 6.1. Let $S$ be a nonabelian simple group different from an alternating group of degree greater than 13. Assume that $S$ is different from $\mathrm{PSL}_{3}(4)$ and $\mathrm{PSU}_{4}(3)$. Let $G$ be a perfect group and $M \triangleleft G$ such that $G / M \cong S,|M| \leq|\operatorname{Mult}(S)|$, and $\operatorname{cd}(G) \subseteq \operatorname{cd}(\operatorname{Schur}(S))$. Then $G$ is uniquely determined up to isomorphism by $S$ and the order of $G$.

Now we prove the main result of this section:

Theorem 6.2. Let $G$ be a finite group and $H$ a perfect central cover of $\mathrm{A}_{6}$ or $A_{7}$. Then $G \cong H$ if and only if $\mathbb{C} G \cong \mathbb{C} H$.

Proof. First, as $\mathrm{A}_{6} \cong \mathrm{PSL}_{2}(9)$, every perfect central cover of $\mathrm{A}_{6}$ can be viewed as a quasisimple classical group, a case which is already studied in [Nguyen 2013, Theorem 1.1]. So it remains to consider the perfect central covers of $\mathrm{A}_{7}$. Let $H$ be one of those, and assume that $G$ is a finite group such that $\mathbb{C} G \cong \mathbb{C} H$.

As before, we see that $G$ is perfect and, if $M$ is a normal maximal subgroup of $G$, we have that $G / M$ is nonabelian simple and $\operatorname{cd}^{*}(G / M) \subseteq \operatorname{cd}^{*}(H)$. In particular,

$$
\operatorname{cd}^{*}(G / M) \subseteq \operatorname{cd}^{*}\left(\operatorname{Schur}\left(\mathrm{A}_{7}\right)\right),
$$


where $\operatorname{Schur}\left(A_{7}\right)$ is the Schur cover (or the 6-fold cover) of $A_{7}$. It follows that $|G / M| \leq 6\left|\mathrm{~A}_{7}\right|=7560$. Inspecting [Conway et al. 1985], we come up with

$$
G / M \cong \mathrm{PSL}_{2}(q) \text { with } 5 \leq q \leq 23 \text {, or } \mathrm{PSL}_{3}(3) \text {, or } \mathrm{PSU}_{3}(3) \text {, or } \mathrm{A}_{7} .
$$

Since each of the possibilities for $G / M$ except $A_{7}$ does not satisfy the inclusion $\operatorname{cd}^{*}(G / M) \subseteq \operatorname{cd}^{*}\left(\operatorname{Schur}\left(\mathrm{A}_{7}\right)\right)$, we deduce that $G / M \cong \mathrm{A}_{7}$.

On the other hand, as $\mathbb{C} G \cong \mathbb{C} H$, we have $|G|=|H|$. It follows that $|M|=$ $|\boldsymbol{Z}(H)| \leq 6$. Using Lemma 6.1, we conclude that $G \cong H$.

\section{Excluding critical character degrees of $\widehat{\mathrm{S}}_{\boldsymbol{n}}$}

In this section, we prove a nonexistence result for special character degrees of $\widehat{S}_{n}$ which will be applied in the next section. Indeed, with the following proposition, we prove a little more, as only the case of even $n$ will be needed (in fact, the proof shows that also versions with slightly modified 2-powers can be obtained).

Proposition 7.1. Let $n \in \mathbb{N}$. If $2^{\left[\frac{n-2}{2}\right]}(n-1)$ is a character degree of $\widehat{\mathrm{S}}_{n}$, then $n \leq 8$ and the degree is an ordinary degree $f_{\lambda}$ for $\lambda \in\left\{(2),(2,1),\left(4,2^{2}\right)\right\}$ (or their conjugates $)$, or the spin degree $g_{\mu}$ for $\mu=(4,2)$.

The strategy for the proof is inspired by the methods used in [Balog et al. 2001; Bessenrodt and Olsson 2002] to classify the irreducible characters of prime power degrees. A main ingredient is a number-theoretic result which is a variation of [Balog et al. 2001, Theorem 3.1].

First, we define $M(n)$ to be the set of pairs of finite sequences of integers $s_{1}<s_{2}<\cdots<s_{r} \leq n, t_{1}<t_{2}<\cdots<t_{r} \leq n$, with all numbers different from $n-1$, that satisfy

(i) $s_{i}<t_{i}$ for all $i$,

(ii) $s_{1}$ and $t_{1}$ are primes $>n / 2$, and

(iii) for $1 \leq i \leq r-1, s_{i+1}$ and $t_{i+1}$ contain prime factors exceeding $2 n-s_{i}-t_{i}$ and not dividing $n-1$.

We then set $t(n):=\max \left\{t_{r} \mid\left(\left(s_{i}\right)_{i=1, \ldots, r},\left(t_{i}\right)_{i=1, \ldots, r}\right) \in M(n)\right\}$, and $t(n)=0$ when $M(n)=\varnothing$. Note that, for all $n \geq 15$, there are at least two primes $p, q \neq n-1$ with $n / 2<p<q \leq n$ (e.g., use [Harborth and Kemnitz 1981]); hence for all $n \geq 15$ the set $M(n)$ is not empty.

Theorem 7.2. Let $n \in \mathbb{N}$. Then $n-t(n) \leq 225$.

For $15 \leq n \leq 10^{9}$, we have the tighter bounds

$$
n-t(n) \begin{cases}=7 & \text { for } n \in\{30,54\}, \\ =5 & \text { for } n \in\{18,24,28,52,102,128,224\} \\ \leq 4 & \text { otherwise. }\end{cases}
$$


Proof. For $n>3.9 \cdot 10^{8}$, the proof follows the lines of the arguments for [Balog et al. 2001, Theorem 3.1], noticing that in the construction given there the numbers in the sequences are below $n-1$ and that the chosen prime factors do not divide $n-1$; this then gives $n-t(n) \leq 225$.

A computer calculation (with Maple) shows that, for all $n \leq 10^{9}$, we have the claimed bounds and values for $n-t(n)$.

For any partition $\lambda$ of $n$, we denote by $l(\lambda)$ the length of $\lambda$, and we let $l_{1}(\lambda)$ be the multiplicity of 1 in $\lambda$. We put $h_{i}=h(i, 1)$ for $1 \leq i \leq l(\lambda)$; these are the first-column hook lengths of $\lambda$. We set $f \operatorname{ch}(\lambda)=\left\{h_{1}, \ldots, h_{l(\lambda)}\right\}$.

First we want to show that Proposition 7.1 holds for ordinary characters. Via computer calculations, the claim is easily checked up to $n=44$, and in particular, we find the stated exceptions for $n<9$. Thus we have to show that

$$
f_{\lambda}=2^{\left[\frac{n-2}{2}\right]}(n-1)
$$

cannot hold for $n \geq 9$; if necessary, we may even assume that $n>44$.

To employ Theorem 7.2, we need some preparation, which is similar to corresponding results in [Balog et al. 2001].

Proposition 7.3. If $q$ is a prime with $n-l_{1}(\lambda) \leq q \leq n$ and $q \nmid f_{\lambda}$, then

$$
q, 2 q, \ldots,\left[\frac{n}{q}\right] q \in \mathrm{fch}(\lambda) .
$$

Proof. Put $w=[n / q], n=w q+r, 0 \leq r<q$. By assumption, we have $(w-1) q \leq$ $(w-1) q+r=n-q \leq l_{1}:=l_{1}(\lambda)$. Thus $q, 2 q, \ldots,(w-1) q \in \operatorname{fch}(\lambda)$. If $w q \leq l_{1}$, then we are done. Assume that $l_{1}<w q$. At most $w$ hooks in $\lambda$ are of lengths divisible by $q$ (see, e.g., [Olsson 1993, Proposition (3.6)]). If there are only the above $w-1$ hooks in the first column of length divisible by $q$, then $q \mid f_{\lambda}$ since $\prod_{i=1}^{w}(i q) \mid n$ !, a contradiction. Let $h(i, j)$ be the additional hook length divisible by $q$. Since $\lambda \neq\left(1^{n}\right), l_{1} \leq h_{2}$. If $h_{2}>l_{1}$, then $h(i, j)+h(2,1)>q+l_{1} \geq n$. By [Balog et al. 2001, Corollary 2.8] we get $j=1$. If $h_{2}=l_{1}$, then $\lambda=\left(n-l_{1}, 1^{l_{1}}\right)$, and since $l_{1}<w q$ there has to be a hook of length divisible by $q$ in the first row. Since $n-l_{1} \leq q$ we must have $h_{1}=w q$.

In analogy to [Balog et al. 2001, Corollary 2.10], we deduce:

Corollary 7.4. Let $1 \leq i<j \leq l(\lambda)$. If $h \leq n$ has a prime divisor $q$ satisfying $2 n-h_{i}-h_{j}<q$ and $q \nmid f_{\lambda}$, then $h \in \operatorname{fch}(\lambda)$.

We now combine these results with Theorem 7.2, similarly to [Balog et al. 2001]; as stated earlier we may assume that $n \geq 15$, and hence there are least two primes $p, q \neq n-1$ with $n / 2<p<q \leq n$. Assuming (*) for $\lambda$, the hook formula implies that there have to be hooks of length $p$ and $q$ in $\lambda$. As argued in [Balog et al. 2001], we then have $p, q \in \mathrm{fch}(\lambda)$ or $p, q \in \mathrm{fch}(\bar{\lambda})$; without loss of generality, we may 
assume $p, q \in \mathrm{fch}(\lambda)$. Then the assumption (*) forces any prime between $n / 2$ and $n$, except $n-1$ if this is prime, to be in $f \operatorname{ch}(\lambda)$. This gives an indication towards the connection with the sequences belonging to the pairs in $M(n)$.

Indeed, we have the following proposition, which is proved similarly to the corresponding result in [Balog et al. 2001].

Proposition 7.5. Let $n \geq 15$. Let $\left(\left(s_{i}\right)_{i=1, \ldots, r},\left(t_{i}\right)_{i=1, \ldots, r}\right) \in M(n)$. Let $\lambda$ be a partition of $n$ such that $(*)$ holds. Then $\left\{s_{1}, \ldots, s_{r}, t_{1}, \ldots, t_{r}\right\} \subset \operatorname{fch}(\lambda)$ or $\left\{s_{1}, \ldots, s_{r}, t_{1}, \ldots, t_{r}\right\} \subset \mathrm{fch}(\bar{\lambda})$.

In particular, $n-h_{1} \leq 225$, and we have tighter bounds for $n-h_{1}$ when $n \leq 10^{9}$ as given in Theorem 7.2.

Now we can embark on the first part of the proof of Proposition 7.1, showing the nonexistence of ordinary irreducible characters of the critical degree for $n \geq 9$. As remarked before, we may assume $n \geq 44$.

First part of the proof of Proposition 7.1. Set $m=[(n-2) / 2]$, and assume that the partition $\lambda$ of $n$ satisfies

$$
f_{\lambda}=2^{m}(n-1)
$$

Let $c=n-h_{1}$. By [Balog et al. 2001, Proposition 4.1] we have the following bound for the 2-part of the degree:

$$
\left(f_{\lambda}\right)_{2} \leq n^{2} \cdot((2 c+2) !)_{2} .
$$

By Proposition 7.5, we have $c \leq 225$, and hence $((2 c+2) !)_{2} \leq(452 !)_{2}=2^{448}$. Thus

$$
2^{m} \leq 2^{448} n^{2} \leq 2^{448}(2 m+3)^{2}
$$

A short computation gives $m \leq 467$, and hence $n \leq 937$. By Proposition 7.5, $c \leq 5$, unless $n=54$, where we only get $c \leq 7$. But for $n=54$ we can argue as follows: As $\lambda$ satisfies $(*)$, without loss of generality $43,47 \in \mathrm{fch}(\lambda)$. Then $l_{1}(\lambda) \geq 35$ (by [Balog et al. 2001, Proposition 2.6]), and hence 17, $34 \in \mathrm{fch}(\lambda)$; since $54>3 \cdot 17$, by the hook formula there has to be one more hook of length divisible by 17 in $\lambda$. As $c \leq 7$, this is in the first row or column; if it is not in the first column, we get a contradiction considering this hook and the one of length 43 . Thus $51 \in \operatorname{fch}(\lambda)$, and hence $c \leq 3$.

Hence for all $n \leq 937$ we have $((2 c+2) !)_{2} \leq(12 !)_{2}=2^{10}$, and

$$
2^{m} \leq 2^{10} n^{2} \leq 2^{10}(2 m+3)^{2} .
$$

This implies $m \leq 20$, and hence $n \leq 43$, where the assertion was checked directly. 
Next we deal with the spin characters of $\widehat{S}_{n}$. Recall that for a bar partition $\lambda$ of $n$, $\bar{g}_{\lambda}$ is the number of shifted standard tableaux of shape $\lambda$, and the spin character degree associated to $\lambda$ is $g_{\lambda}=2^{\left[\frac{n-l(\lambda)}{2}\right]} \bar{g}_{\lambda}$. Hence the condition on the spin degree translates into the condition $(\dagger)$ on $\bar{g}_{\lambda}$ given below.

Proposition 7.6. Let $\lambda$ be a bar partition of $n$. Then

$$
\bar{g}_{\lambda}= \begin{cases}2^{\left\lceil\frac{l(\lambda)-2}{2}\right\rceil}(n-1) & \text { for } n \text { even }, \\ 2^{\left\lceil\frac{l(\lambda)-3}{2}\right\rceil}(n-1) & \text { for } n \text { odd }\end{cases}
$$

only if $n \leq 6$ and $\lambda=(2), \lambda=(4,2)$.

We note that, for $n \leq 34$, the assertion is easily checked by computer calculation (using John Stembridge's Maple package QF), so we may assume that $n>34$ when needed.

We set $b_{i}=b(1, i)$ for the first row bar lengths of $\lambda$, and $\operatorname{frb}(\lambda)$ for the set of first row bar lengths of $\lambda$ (see [Olsson 1993] for details on the combinatorics of bars).

In analogy to the case of ordinary characters where we have modified the results in [Balog et al. 2001], we adapt the results in [Bessenrodt and Olsson 2002] for the case under consideration now. Similarly to Proposition 7.3 before, we have a version of [Bessenrodt and Olsson 2002, Proposition 2.5] where, instead of the prime power condition for $\bar{g}_{\lambda}$, the condition $q \nmid \bar{g}_{\lambda}$ is assumed for the prime $q$ under consideration. For the corresponding variant of [Bessenrodt and Olsson 2002, Lemma 2.6] that says that any prime $q$ with $n / 2<q \leq n$ and $q \nmid \bar{g}_{\lambda}$ is a first row bar length of $\lambda$, we need two primes $p_{1}, p_{2} \neq n-1$ with $p_{1}+p_{2}-n>n / 2$. For $n \geq 33, n \neq 42$, we always find two primes $p_{1}, p_{2} \neq n-1$ such that $\frac{3}{4} n<p_{1}<p_{2} \leq n$. But for $n=42$, the primes $p_{1}=31$ and $p_{2}=37$ are big enough to have $p_{1}+p_{2}-n>n / 2$.

The largest bar length of $\lambda$ is $b_{1}=b(1,1)=\lambda_{1}+\lambda_{2}$. As before, the preparatory results just described together with our arithmetical Theorem 7.2 show that $n-b_{1}$ is small for a bar partition $\lambda$ satisfying $(\dagger)$. More precisely, we obtain:

Proposition 7.7. Let $n \geq 15$. Let $\left(\left(s_{1}, \ldots, s_{r}\right),\left(t_{1}, \ldots, t_{r}\right)\right) \in M(n)$. Assume that $\lambda$ is a bar partition of $n$ that satisfies $(\dagger)$. Then $s_{1}, \ldots, s_{r}, t_{1}, \ldots, t_{r} \in \operatorname{frb}(\lambda)$.

In particular, if $\lambda$ satisfies $(\dagger)$, then $n-b_{1} \leq 225$, and we have tighter bounds for $n-b_{1}$ when $n \leq 10^{9}$ as given in Theorem 7.2.

Now we can get into the second part of the proof of Proposition 7.1, showing the nonexistence of spin irreducible characters of the critical degree for $n \geq 7$. 
Second part of the proof of Proposition 7.1. We have already seen that it suffices to prove Proposition 7.6, and that we may assume $n \geq 15$. Set

$$
r= \begin{cases}\left\lceil\frac{l(\lambda)-2}{2}\right\rceil & \text { for } n \text { even, } \\ \left\lceil\frac{l(\lambda)-3}{2}\right\rceil & \text { for } n \text { odd }\end{cases}
$$

and assume that $\lambda$ is a bar partition of $n$ that satisfies $(\dagger)$.

Let $c=n-b_{1}$. As seen above, we have $c \leq 225$, and hence $l(\lambda) \leq 23$. Thus $r \leq 11$ in any case, and hence $\bar{g}_{\lambda} \leq 2^{11}(n-1)$.

Now, by [Bessenrodt and Olsson 2002, Proposition 2.2] we know that $\bar{g}_{\lambda} \geq$ $\frac{1}{2}(n-1)(n-4)$ unless we have one of the following situations: $\lambda=(n)$ and $\bar{g}_{\lambda}=1$, or $\lambda=(n-1,1)$ and $\bar{g}_{\lambda}=n-2$. None of these exceptional cases is relevant here, and thus we obtain $n-4 \leq 2^{12}$. But for $n \leq 4100$ we already know that $c \leq 7$. Then $l(\lambda) \leq 6$ and $r \leq 2$, and hence

$$
\frac{1}{2}(n-1)(n-4) \leq \bar{g}_{\lambda} \leq 4(n-1) .
$$

But then $n-4 \leq 8$, a contradiction. Thus we have now completed the proof of Proposition 7.1.

\section{Eliminating simple groups of Lie type in even characteristic}

Let $G$ be a finite group whose complex group algebra is isomorphic to that of $\widehat{S}_{n}$. In order to show that $G$ is isomorphic to one of the two double covers of $\mathrm{S}_{n}$, one has to eliminate the involvement of all nonabelian simple groups other than $A_{n}$ in the structure of $G$. The most difficult case turns out to be the simple groups of Lie type in even characteristic.

For the purpose of the next lemma, let $\mathscr{b}$ be the set consisting of the following simple groups:

$$
\begin{aligned}
& \left\{{ }^{2} F_{4}(2)^{\prime}, \mathrm{PSL}_{4}(2), \mathrm{PSL}_{3}(4), \mathrm{PSU}_{4}(2), \mathrm{PSU}_{6}(2),\right. \\
& \left.\qquad \mathrm{P}_{8}^{+}(2), \mathrm{PSp}_{6}(2),{ }^{2} B_{2}(8), G_{2}(4),{ }^{2} E_{6}(2)\right\} .
\end{aligned}
$$

Lemma 8.1. If $S$ is a simple group of Lie type in characteristic 2 such that $|S|_{2} \geq 2^{4}$ and $S \notin \mathscr{C}$, then $|S|_{2}<2^{(e(S)-1) / 2}$, where $e(S)$ is the smallest nontrivial degree of an irreducible projective representation of $S$.

Proof. Assume that $S$ is defined over a finite field of size $q=2^{f}$. If $|S|_{2}=q^{N(S)}$, then the inequality in the lemma is equivalent to

$$
e(S)>2 N(S) f+1 \text {. }
$$


The values of $e(S)$ are available in [Tiep and Zalesskii 1996, Table II] for classical groups and in [Lübeck 2001] for exceptional groups. The arguments for simple classical groups are quite similar. So let us consider the linear groups. Assume that $S=\operatorname{PSL}_{m}(q)$ with $m \geq 2$. We have $N(S)=m(m-1) / 2$. First we assume that $m=2$. As $|S|_{2}=q \geq 2^{4}$, we deduce that $e(S)=q-1$ and $f \geq 4$. In this case, we obtain $e(S)-1=2^{f}-2$ and $2 N(S) f+1=2 f+1$. As $f \geq 4$, we see that (8-1) holds. Next we assume that $m \geq 3$ and $S \neq \mathrm{PSL}_{3}$ (4), $\mathrm{PSL}_{4}(2)$. As $|S|_{2} \geq 2^{4}$, we deduce that $S \neq \mathrm{PSL}_{3}(2)$. We have $e(S)=\left(q^{m}-q\right) /(q-1)$. Now (8-1) is equivalent to

$$
\frac{q^{m}-q}{q-1}>m(m-1) f+1
$$

It is routine to check that this inequality holds for any $m \geq 3$ and $q \geq 2$.

The arguments for exceptional Lie type groups are also similar. For instance, if $S={ }^{2} B_{2}\left(2^{2 m+1}\right)$ with $m \geq 1$, then $|S|_{2}=2^{2(2 m+1)}$ and $e(S)=2^{m}\left(2^{2 m+1}-1\right)$. The inequality can now be easily checked.

Proposition 8.2. Let $G$ be a finite group and $S$ a simple group of Lie type in characteristic 2. Suppose that $M \subseteq G^{\prime}$ is a normal subgroup of $G$ such that $G^{\prime} / M \cong S$ and $\left|G: G^{\prime}\right|=2$. Then $\operatorname{cd}^{*}(G) \neq \operatorname{cd}^{*}\left(\widehat{\mathrm{S}}_{n}\right)$ for every integer $n \geq 10$.

Proof. By way of contradiction, assume that $\operatorname{cd}^{*}(G)=\operatorname{cd}^{*}\left(\widehat{S}_{n}\right)$ for some $n \geq 10$. Let $\mathrm{St}_{S}$ be the Steinberg character of $G^{\prime} / M \cong S$. As $\mathrm{St}_{S}$ extends to $G / M$ and $\left|G / M: G^{\prime} / M\right|=2$, by Gallagher's theorem (see [Isaacs 1994, Corollary 6.17] for instance) $G / M$ has two irreducible characters of degree $\operatorname{St}_{S}(1)=|S|_{2}$. As $n \geq 10$, Lemma 3.5(1) yields that $d_{1}\left(\widehat{\mathrm{S}}_{n}\right)=n-1$.

We claim that $|S|_{2}>d_{1}\left(\widehat{\mathrm{S}}_{n}\right)=n-1$. Suppose for a contradiction that $|S|_{2}=$ $d_{1}\left(\widehat{\mathrm{S}}_{n}\right)$. If $G / M \cong S \times C_{2}$, then $\operatorname{cd}(G / M)=\operatorname{cd}(S) \subseteq \operatorname{cd}\left(\widehat{\mathrm{S}}_{n}\right)$, which implies that $d_{1}(S) \geq d_{1}\left(\widehat{\mathrm{S}}_{n}\right)=|S|_{2}$. However, this is impossible since $S$ always has a nontrivial character degree smaller than $|S|_{2}$ (see [Tong-Viet 2012, Lemma 8] for instance). Now assume that $G / M$ is almost simple with socle $S$. If $S \not \operatorname{PSL}_{2}(q)$ with $q \geq 4$, then $d_{1}(G / M)<|S|_{2}=d_{1}\left(\widehat{\mathrm{S}}_{n}\right)$ by [Tong-Viet 2011, Lemma 2.4], which leads to a contradiction as before since $\operatorname{cd}(G / N) \subseteq \operatorname{cd}\left(\widehat{\mathrm{S}}_{n}\right)$. Therefore, assume that $S=\operatorname{PSL}_{2}(q)$ with $q=2^{f} \geq 4$. Then $q=|S|_{2}=n-1 \geq 9$. If $q \equiv-1(\bmod 3)$, then $d_{1}(G)=q-1<q=d_{1}\left(\widehat{\mathrm{S}}_{n}\right)$ by [Tong-Viet 2011, Lemma 2.5], which is impossible. Hence, $q \equiv 1(\bmod 3)$ and $q+1 \in \operatorname{cd}(G / M) \subseteq$ $\operatorname{cd}\left(\widehat{\mathrm{S}}_{n}\right)$. It follows that $q+1=(n-1)+1=n \in \operatorname{cd}\left(\widehat{\mathrm{S}}_{n}\right)$. If $n \geq 12$, then $d_{2}\left(\widehat{\mathrm{S}}_{n}\right)>2 n>n>d_{1}\left(\widehat{\mathrm{S}}_{n}\right)$, hence $n$ is not a degree of $\widehat{\mathrm{S}}_{n}$. Thus $10 \leq n \leq 11$. However, we see that $n-1$ is not a power of 2 in either case. The claim is proved. 
Assume that $n=2 k+1 \geq 11$ is odd. By Lemmas 2.1 and $2.3,|S|_{2}=2^{k}$ is the degree of the basic spin character of $\widehat{S}_{n}$. However, by [Wales 1979, Table I] such a degree has multiplicity 1 , which contradicts the fact proved above that $G$ has at least two irreducible characters of degree $|S|_{2}$.

Assume $n=2 k \geq 10$ is even. By Lemmas 2.1 and 2.3 and [Wales 1979, Table I], $\widehat{\mathrm{S}}_{n}$ always has the character degree $2^{k-1}$ with multiplicity 2 , and, if $n=2^{r}+2$, then it has the character degree $2^{k-1}(n-2)=2^{2^{r-1}+r}$ with multiplicity 1 . These are in fact the only nontrivial 2-power character degrees of $\widehat{S}_{n}$. As in the previous case, by comparing the multiplicity, we see that $|S|_{2} \neq 2^{2^{r-1}+r}$. Thus $|S|_{2}=2^{k-1}$ is the degree of the basic spin character of $\widehat{S}_{n}$ with multiplicity 2 . Notice that $k \geq 5$ and hence $|S|_{2}=2^{k-1} \geq 2^{4}$.

Now let $\psi \in \operatorname{Irr}(G)$ with $\psi(1)=n-1$. As $\left|G: G^{\prime}\right|=2$ and $\psi(1)$ is odd, we deduce that $\phi=\psi \downarrow_{G^{\prime}} \in \operatorname{Irr}\left(G^{\prime}\right)$ and $\phi(1)=n-1$. Let $\theta \in \operatorname{Irr}(M)$ be an irreducible constituent of $\phi \downarrow_{M}$. Then $\phi \downarrow_{M}=e\left(\theta_{1}+\cdots+\theta_{t}\right)$, where $t=\left|G^{\prime}: I_{G^{\prime}}(\theta)\right|$, and each $\theta_{i}$ is conjugate to $\theta \in \operatorname{Irr}(M)$. If $\theta$ is not $G^{\prime}$-invariant, then $\phi(1)=$ $\operatorname{et} \theta(1)=n-1 \geq \min (S)$, where $\min (S)$ is the smallest nontrivial index of a maximal subgroup of $S$. We see that $\min (S)>d_{1}(S) \geq e(S)$, where $e(S)$ is the minimal degree of a projective irreducible representation of $S$, and so $n-1 \geq e(S)$. If $\theta$ is $G^{\prime}$-invariant and $\phi \downarrow M=e \theta$ with $e>1$, then $e$ is the degree of a projective irreducible representation of $S$. It follows that $n-1 \geq e \geq e(S)$. In both cases, we always have

$$
k-1=\frac{n-2}{2} \geq \frac{e(S)-1}{2} .
$$

Therefore,

$$
|S|_{2}=2^{k-1} \geq 2^{(e(S)-1) / 2} .
$$

By Lemma 8.1, we deduce that $S \in \mathscr{C}$. Solving the equation $|S|_{2}=2^{(n-2) / 2}$, we get the degree $n$. However, by using [Conway et al. 1985] and Lemma 3.5, we can check that $\operatorname{cd}(G / M) \nsubseteq \mathrm{cd}\left(\widehat{\mathrm{S}}_{n}\right)$ in any of these cases. For example, assume that $S \cong{ }^{2} E_{6}(2)$. Then $|S|_{2}=2^{36}=2^{(n-2) / 2}$, so $n=74$. By Lemma 3.5, we have $d_{1}\left(\widehat{\mathrm{S}}_{n}\right)=n-1=73$ and $d_{2}\left(\widehat{\mathrm{S}}_{n}\right)=n(n-3) / 2=2627$. Using [Conway et al. 1985], we know that $\operatorname{cd}(G / M)$ contains the degree 1938. Clearly, $d_{1}\left(\widehat{\mathrm{S}}_{n}\right)<1938<d_{2}\left(\widehat{\mathrm{S}}_{n}\right)$, so $1938 \notin \operatorname{cd}\left(\widehat{\mathrm{S}}_{n}\right)$, hence $\operatorname{cd}(G / M) \nsubseteq \operatorname{cd}\left(\widehat{\mathrm{S}}_{n}\right)$, a contradiction.

Finally we assume that $e t=1$. Then $\theta$ extends to $\phi \in \operatorname{Irr}\left(G^{\prime}\right)$ and to $\psi \in \operatorname{Irr}(G)$. Hence $\phi \downarrow M=\theta$ and so, by Gallagher's Theorem, we have $\psi \tau \in \operatorname{Irr}(G)$ for every $\tau \in \operatorname{Irr}(G / M)$. In particular,

$$
2^{k-1}(n-1)=\psi(1)|S|_{2} \in \operatorname{cd}(G)=\operatorname{cd}\left(\widehat{S}_{n}\right),
$$

which is impossible by Proposition 7.1. 


\section{Eliminating simple groups other than $A_{n}$}

We continue to eliminate the involvement of simple groups other than $A_{n}$ in the structure of $G$ with $\mathbb{C} G \cong \mathbb{C} \widehat{S}_{n}$.

Proposition 9.1. Let $G$ be an almost-simple group with nonabelian simple socle $S$. Suppose that $\operatorname{cd}^{*}(G) \subseteq \operatorname{cd}^{*}\left(\widehat{\mathrm{S}}_{n}\right)$ for some $n \geq 10$. Then $S \cong \mathrm{A}_{n}$ or $S$ is isomorphic to a simple group of Lie type in characteristic 2.

Proof. We make use of the classification of finite simple groups.

(i) $S$ is a sporadic simple group or the Tits group. Using GAP (version 4.4.12), we can assume that $n \geq 19$. By Lemma 3.5(2), we have $d_{2}\left(\widehat{\mathrm{S}}_{n}\right)=n(n-3) / 2 \geq 152$. Since $d_{2}(G) \geq d_{2}\left(\widehat{\widehat{S}}_{n}\right) \geq 152$, using [Conway et al. 1985], we only need to consider the following simple groups:

$J_{3}$, Suz, McL, Ru, He, $\mathrm{Co}_{1}, \mathrm{Co}_{2}, \mathrm{Co}_{3}, \mathrm{Fi}_{22}, \mathrm{O}^{\prime} \mathrm{N}, \mathrm{HN}, \mathrm{Ly}, \mathrm{Th}, \mathrm{Fi}_{23}, J_{4}, \mathrm{Fi}_{24}^{\prime}, B, M$.

To eliminate these groups, we first observe that $n \geq p(S)$, the largest prime divisor of $|S|$, and $d_{i}(G) \geq d_{i}\left(\widehat{\mathrm{S}}_{n}\right)$ for all $i \geq 1$. Now with the lower bound $n \geq \max \{19, p(S)\}$, we can find the lower bounds for $d_{i}\left(\widehat{\mathrm{S}}_{n}\right)$ with $1 \leq i \leq 7$ using Lemmas 3.4 and 3.5. Choose $i \in\{2,3, \ldots, 7\}$ such that $d_{i}\left(\widehat{\mathrm{S}}_{n}\right)>\bar{d}_{j}(G)$ for some $j \geq 1$ such that $|i-j|$ is minimal. If $i \geq j$, then we obtain a contradiction. Otherwise, $d_{j}(G) \in$ $\left\{d_{k}\left(\widehat{S}_{n}\right)\right\}_{k=j}^{i-1}$. Solving these equations for $n$, we then obtain that either these equations have no solution, or that, for each solution of $n$, we can find some $k \geq 1$ with $d_{k}\left(\widehat{\mathrm{S}}_{n}\right)>d_{k}(G)$.

For an example of such a demonstration, assume that $S=\mathrm{O}^{\prime} \mathrm{N}$. In this case, we have $|\operatorname{Out}(S)|=2$, so $G=S$ or $G=S$.2. Since $p(S)=31$, we have $n \geq 31$. Assume first that $G=S=\mathrm{O}^{\prime} \mathrm{N}$. Then $d_{4}\left(\mathrm{O}^{\prime} \mathrm{N}\right)=26752$ and, since $n \geq 31$, by Lemma 3.4 $d_{7}\left(\widehat{\mathrm{S}}_{n}\right) \geq 26970>d_{4}\left(\mathrm{O}^{\prime} \mathrm{N}\right)$. It follows that $d_{4}\left(\mathrm{O}^{\prime} \mathrm{N}\right) \in\left\{d_{i}\left(\hat{\mathrm{S}}_{n}\right)\right\}_{i=4}^{6}$. However, we can check that these equations are impossible. Now assume $G=\mathrm{O}^{\prime} \mathrm{N}$.2. Then $d_{2}(G)=26752<26970 \leq d_{7}\left(\widehat{\mathrm{S}}_{n}\right)$ so that $d_{2}(G) \in\left\{d_{i}\left(\widehat{\mathrm{S}}_{n}\right)\right\}_{i=2}^{6}$. As above, these equations cannot hold for any $n \geq 31$. Thus $\operatorname{cd}(G) \nsubseteq \operatorname{cd}\left(\widehat{\mathrm{S}}_{n}\right)$.

For another example, let $S=M$. Since $|\operatorname{Out}(S)|=1$, we have $G=S$ so that $p(S)=71 \in \pi\left(\widehat{\mathrm{S}}_{n}\right)$ and hence $n \geq 71$. As $d_{1}(M)=196883<914480 \leq d_{7}\left(\widehat{\mathrm{S}}_{n}\right)$, we deduce that $d_{1}(M) \in\left\{d_{i}\left(\widehat{\mathrm{S}}_{n}\right) \mid i=1, \ldots, 6\right\}$. Solving these equations, we obtain $n=196884$. But then $d_{2}\left(\widehat{S}_{n}\right)>21296876=d_{2}(M)$. Thus $\operatorname{cd}(M) \nsubseteq \operatorname{cd}\left(\widehat{\mathrm{S}}_{n}\right)$.

(ii) $S=\mathrm{A}_{m}$ with $m \geq 7$. Note that we consider $\mathrm{A}_{5} \cong \mathrm{PSL}_{2}$ (5) and $\mathrm{A}_{6} \cong \mathrm{PSL}_{2}$ (9) as groups of Lie type. Let $\lambda=(m-1,1)$, a partition of $m$. Since $m \geq 7, \lambda$ is not self-conjugate, hence the irreducible character $\chi_{\lambda}$ of $S_{m}$ is still irreducible upon restriction to $\mathrm{A}_{m}$. Note that $\operatorname{Aut}\left(\mathrm{A}_{m}\right)=\mathrm{S}_{m}$ as $m \geq 7$. Then $G \in\left\{\mathrm{A}_{m}, \mathrm{~S}_{m}\right\}$ and $G$ has an irreducible character of degree $m-1$. Since $\operatorname{cd}(G) \subseteq \operatorname{cd}\left(\widehat{\mathrm{S}}_{n}\right)$, we have $m-1 \geq d_{1}\left(\widehat{\mathrm{S}}_{n}\right)=n-1$, so $m \geq n$. If $m=n$ then we are done. On the other hand, 
if $m>n$ then

$$
|G| \geq|S|=\left|\mathrm{A}_{m}\right|>4\left|\mathrm{~A}_{n}\right|=\left|\widehat{\mathrm{S}}_{n}\right|,
$$

and this violates the hypothesis $\operatorname{cd}^{*}(G) \subseteq \operatorname{cd}^{*}\left(\widehat{\mathrm{S}}_{n}\right)$.

(iii) $S$ is a simple group of Lie type in odd characteristic. Suppose that $S=G\left(p^{k}\right)$, a simple group of Lie type defined over a field of $p^{k}$ elements with $p$ odd. Let $\mathrm{St}_{S}$ be the Steinberg character of $S$. Then, as St extends to $G$ and $\operatorname{St}_{S}(1)=|S|_{p}$, we have $|S|_{p} \in \operatorname{cd}\left(\widehat{\mathrm{S}}_{n}\right)$. Using Lemma 2.3, which says that all possible prime power degrees of spin characters of $\mathrm{S}_{n}$ are even, we deduce that $|S|_{p} \in \operatorname{cd}\left(\mathrm{S}_{n}\right)$. By Lemma 2.1, we then obtain that $|S|_{p}=n-1$ since $n \geq 10$. By Lemma 3.5, $n-1=d_{1}\left(\widehat{\mathrm{S}}_{n}\right)$ is the smallest nontrivial degree of $\widehat{\mathrm{S}}_{n}$. Assume first that $S \neq \operatorname{PSL}_{2}(q)$. Then $d_{1}(G)<|S|_{p}=d_{1}\left(\widehat{\mathrm{S}}_{n}\right)$ by [Tong-Viet 2011, Lemma 2.4], which is a contradiction as $\operatorname{cd}(G) \subseteq \operatorname{cd}\left(\widehat{\mathrm{S}}_{n}\right)$. Now it remains to consider the case $S=\operatorname{PSL}_{2}(q)$. We have $q=n-1 \geq 9$. If $G$ has a character degree which is smaller than $|S|_{p}=q$, then we obtain a contradiction as before. So, by [Tong-Viet 2011, Lemma 2.5], we have $p \neq 3$ and $q \equiv 1(\bmod 3)$ or $p=3$ and $q \equiv 1(\bmod 4)$. In both cases, $G$ has an irreducible character of degree $q+1=n=d_{1}\left(\widehat{\mathrm{S}}_{n}\right)+1$. If $n \geq 12$, then $d_{2}\left(\widehat{\mathrm{S}}_{n}\right) \geq 2 n>n>d_{1}\left(\widehat{\mathrm{S}}_{n}\right)$ by Lemma 3.5 , so that $n$ is not a character degree of $\widehat{\mathrm{S}}_{n}$. Assume that $10 \leq n \leq 11$. Then $n=10$ and $q=9$. However, using [Conway et al. 1985], we can check that $\operatorname{cd}(G) \nsubseteq \subseteq \operatorname{cd}\left(\widehat{\mathrm{S}}_{n}\right)$ for every almost-simple group $G$ with socle $\mathrm{PSL}_{2}(9) \cong \mathrm{A}_{6}$.

Combining Propositions 9.1 and 8.2, we obtain the following results, which will be crucial in the proof of Theorem A:

Proposition 9.2. Let $G$ be a finite group and let $M \subseteq G^{\prime}$ be a normal subgroup of $G$ such that $G / M$ is an almost-simple group with socle $S \neq \mathrm{A}_{n}$, where $\left|G: G^{\prime}\right|=2$ and $G^{\prime} / M \cong S$. Then $\operatorname{cd}^{*}(G) \neq \operatorname{cd}^{*}\left(\widehat{\mathrm{S}}_{n}\right)$.

Proof. If $n \geq 10$, then the result follows from Propositions 9.1 and 8.2. It remains to assume that $5 \leq n \leq 9$ and suppose by contradiction that $\operatorname{cd}^{*}(G)=\operatorname{cd}^{*}\left(\widehat{\mathrm{S}}_{n}\right)$. Then $|G|=2 n$ ! and so $|S| \mid 2 n$ !, hence $\pi(S) \subseteq \pi\left(\widehat{\mathrm{S}}_{n}\right) \subseteq\{2,3,5,7\}$. By [Huppert and Lempken 2000, Theorem III], one of the following holds:

(1) If $\pi(S)=\{2,3,5\}$, then $S \cong \mathrm{A}_{5}, \mathrm{~A}_{6}$ or $\mathrm{PSp}_{4}(3)$.

(2) If $\pi(S)=\{2,3,7\}$, then $S \cong \mathrm{PSL}_{2}$ (7), $\mathrm{PSL}_{2}$ (8) or $\mathrm{PSU}_{3}(3)$.

(3) If $\pi(S)=\{2,3,5,7\}$, then $S \cong \mathrm{A}_{k}$ with $7 \leq k \leq 10, J_{2}, \mathrm{PSL}_{2}(49), \mathrm{PSL}_{3}(4)$, $\mathrm{PSU}_{3}(5), \mathrm{PSU}_{4}(3), \mathrm{PSp}_{4}(7), \mathrm{PSp}_{6}(2)$ or $\mathrm{P}_{8}^{+}(2)$.

Now it is routine to check that $\operatorname{cd}(G / M) \nsubseteq \operatorname{cd}\left(\widehat{\mathrm{S}}_{n}\right)$ unless $S \cong \mathrm{A}_{n}$, where $G / M$ is almost simple with socle $S$. 
Proposition 9.3. Let $G$ be a finite group and let $M \subseteq G^{\prime}$ be a normal subgroup of $G$. Suppose that $\operatorname{cd}^{*}(G)=\operatorname{cd}^{*}\left(\widehat{\mathrm{S}}_{n}\right)$ and $G / M \cong G^{\prime} / M \times C_{2} \cong S^{k} \times C_{2}$ for some positive integer $k$ and some nonabelian simple group $S$. Then $k=1$ and $S \cong \mathrm{A}_{n}$.

Proof. Since $\operatorname{cd}^{*}(S) \subseteq \mathrm{cd}^{*}\left(S^{k}\right)$, the hypotheses imply that

$$
\operatorname{cd}^{*}(S) \subseteq \operatorname{cd}^{*}\left(\widehat{S}_{n}\right)
$$

Assume first that $5 \leq n \leq 9$. Since $\left|S^{k}\right|=|S|^{k}$ divides $\left|\widehat{S}_{n}\right|=2 n$ !, we deduce that $\pi(S) \subseteq \pi\left(\widehat{\mathrm{S}}_{n}\right)$ and, in particular, $\pi(S) \subseteq\{2,3,5,7\}$. The possibilities for $S$ are listed in the proof of Proposition 9.2 above. Observe that $|S|$ is always divisible by a prime $r$ with $r \geq 5$. Hence, $r^{k}|| \widehat{S}_{n} \mid$, which implies that $k=1$ as $\left|\widehat{S}_{n}\right|$ divides $2 \cdot 9$ !. Now the fact that $S \cong \mathrm{A}_{n}$ follows easily.

From now on we can assume that $n \geq 10$. Using Proposition 9.1, we obtain $S=\mathrm{A}_{n}$ or $S$ is a simple group of Lie type in even characteristic. It suffices to show that $k=1$, and then the result follows from Proposition 8.2.

Assume that the latter case holds. Then $S$ is a simple group of Lie type in characteristic 2. By Lemmas 2.1 and 2.3, $\widehat{\mathrm{S}}_{n}$ has at most two distinct nontrivial 2power character degrees, which are $n-1$ and $2^{\lfloor(n-1) / 2\rfloor}$, or $2^{\lfloor(n-1) / 2\rfloor}$ and $2^{2^{r-1}+r}$ with $n=2^{r}+2$. By way of contradiction, assume that $k \geq 2$. If $k \geq 3$, then $G / M \cong S^{k} \times C_{2}$ has irreducible characters of degrees

$$
|S|_{2}^{k}>|S|_{2}^{k-1}>|S|_{2}^{k-2}>1 \text {. }
$$

Obviously, this is impossible as $\operatorname{cd}(G / M) \subseteq \operatorname{cd}\left(\widehat{\mathrm{S}}_{n}\right)$. Therefore, $k=2$. In this case, $G / M$ has character degree $|S|_{2}^{2}$ with multiplicity at least 2 and $|S|_{2}$ with multiplicity at least 4 . It follows that either $2^{\lfloor(n-1) / 2\rfloor}=|S|_{2}^{2}$ and $n-1=|S|_{2}$, or $2^{2^{r-1}+r}=|S|_{2}^{2}$ and $2^{\lfloor(n-1) / 2\rfloor}=|S|_{2}$. However, both cases are impossible by comparing the multiplicity.

It remains to eliminate the case $S \cong \mathrm{A}_{n}$ and $k \geq 2$. By comparing the orders, we see that

$$
2(n ! / 2)^{k}|M|=2 n !
$$

After simplifying, we obtain

$$
|M|(n !)^{k-1}=2^{k} .
$$

Since $n \geq 10$, we see that, if $k \geq 2$, then the left side is divisible by 5 , while the right side is not. We conclude that $k=1$, and the proof is now complete.

\section{Completion of the proof of Theorem $C$}

We need one more result before proving Theorem $\mathrm{C}$. 
Proposition 10.1. Let $G$ be a finite group and let $S$ be a nonabelian simple group. Suppose that $\left|G: G^{\prime}\right|=2$ and $G^{\prime} \cong S^{2}$ is the unique minimal normal subgroup of $G$. Then $\operatorname{cd}(G) \nsubseteq \operatorname{cd}\left(\widehat{\mathrm{S}}_{n}\right)$.

Proof. Assume, to the contrary, that $\operatorname{cd}(G) \subseteq \operatorname{cd}\left(\widehat{\mathrm{S}}_{n}\right)$. Let $\alpha \in \operatorname{Irr}(S)$ with $\alpha(1)>1$ and put $\theta=\alpha \otimes 1 \in \operatorname{Irr}\left(G^{\prime}\right)$. Observe that $\theta$ is not $G$-invariant, so that $I_{G}(\theta)=G^{\prime}$; hence $\theta^{G} \in \operatorname{Irr}(G)$ and so $\theta^{G}(1)=2 \alpha(1) \in \operatorname{cd}\left(\widehat{\mathrm{S}}_{n}\right)$. On the other hand, if $\varphi=$ $\alpha \otimes \alpha \in \operatorname{Irr}\left(G^{\prime}\right)$, then $\varphi$ is $G$-invariant and, since $G / G^{\prime}$ is cyclic, we deduce that $\varphi$ extends to $\psi \in \operatorname{Irr}(G)$, so $\psi(1)=\alpha(1)^{2} \in \operatorname{cd}\left(\widehat{\mathrm{S}}_{n}\right)$. Thus, we conclude that

$$
\text { if } a \in \operatorname{cd}(S) \backslash\{1\} \quad \text { then } 2 a, a^{2} \in \operatorname{cd}\left(\widehat{\mathrm{S}}_{n}\right) .
$$

Let $r$ be an odd prime divisor of $|S|$. The Ito-Michler theorem then implies that $r$ divides some character degree, say $a$, of $S$. Since $a^{2} \in \operatorname{cd}\left(\widehat{S}_{n}\right)$ by (10-1), we have $r^{2} \mid 2 n$ ! and hence $n \geq 2 r$ as $r>2$. Thus, we have shown that

$$
\text { if } r \in \pi(S)-\{2\} \quad \text { then } r^{2} \mid 2 n ! \text { and } n \geq 2 r \text {. }
$$

Using the classification of finite simple groups, we consider the following cases: (i) $S=\mathrm{A}_{m}$, with $m \geq 7$. As $7 \in \pi(S)$, it follows from (10-2) that $n \geq 14$. Since $m-1 \in \operatorname{cd}(S)$, both $2(m-1)$ and $(m-1)^{2}$ are in $\operatorname{cd}\left(\hat{\mathrm{S}}_{n}\right)$ by (10-1). As $m \geq 7$, we also have that $m(m-3) / 2,(m-1)(m-2) / 2 \in \operatorname{cd}(S)$ and so $m(m-3),(m-1)(m-2) \in$ $\operatorname{cd}\left(\widehat{\mathrm{S}}_{n}\right)$. We claim that $m<n$. Suppose for a contradiction that $m \geq n$. As $n \geq 14$, by Lemma 4.1, there exists a prime $r$ such that $n / 2<r \leq n$. Hence, the $r$-part of $2 n$ ! is just $r$. However, as $r \leq n \leq m, r$ divides $\left|\mathrm{A}_{m}\right|$ and so $r^{2} \mid 2 n$ ! by (10-2), a contradiction. Thus $m<n$ as claimed.

Since $m \geq 7$, we obtain that

$$
1<2(m-1)<m(m-3)<(m-1)(m-2)<(m-1)^{2} .
$$

By Lemma 3.5(1), we have $d_{1}\left(\widehat{\mathrm{S}}_{n}\right)=n-1$, so $2(m-1) \geq n-1$, and thus $n \leq 2 m-1$. As $n \geq 14$, we deduce that $m \geq 8$.

Assume first that $m \in\{8,9,10\}$. Then $(m-1)^{2} \in \operatorname{cd}\left(\widehat{S}_{n}\right)$ is a prime power. As $3^{3} \neq(m-1)^{2}>d_{1}\left(\widehat{S}_{n}\right)$, Lemmas 2.1 and 2.3 yield that $(m-1)^{2}$ is a power of 2 , and thus $m=9$. Since $\left\{2^{3}, 3^{3}\right\} \subseteq \operatorname{cd}\left(\mathrm{A}_{9}\right)$, we have $\left\{2^{4}, 2^{6}, 3^{6}\right\} \subseteq \operatorname{cd}\left(\widehat{\mathrm{S}}_{n}\right)$ by $(10-2)$. As $n \geq 14$, we have $2^{\lfloor(n-1) / 2\rfloor}>2^{4}$ and $n(n-3) / 2>2^{4}$, so $d_{2}\left(\widehat{\mathrm{S}}_{n}\right)>2^{4}$ by Lemma 3.5(2). This forces $2^{4}=d_{1}\left(\widehat{S}_{n}\right)=n-1$ or, equivalently, $n=17$. But then Lemmas 2.1 and 2.3 yield $3^{6}=d_{1}\left(\widehat{\mathrm{S}}_{n}\right)$, which is impossible.

Assume next that $m \geq 11$. Then $n \geq 22$ by (10-2). By Lemma 3.5(2), we have $d_{2}\left(\widehat{\mathrm{S}}_{n}\right)>2 n>2 m$. In particular, $2(m-1)<d_{2}\left(\widehat{\mathrm{S}}_{n}\right)$. By $(10-1)$, we have $2(m-1) \in$ $\operatorname{cd}\left(\widehat{\mathrm{S}}_{n}\right)$, hence $2(m-1)=d_{1}\left(\widehat{\mathrm{S}}_{n}\right)=n-1$, which implies that $n=2 m-1$. By Lemma 3.5(3), we have $d_{3}\left(\widehat{\mathrm{S}}_{n}\right)=(n-1)(n-2) / 2$ and thus by (10-3) we obtain that

$$
(m-1)(m-2) \geq d_{3}\left(\widehat{\mathrm{S}}_{n}\right)=(n-1)(n-2) / 2=(m-1)(2 m-3) .
$$


After simplifying, we have $m-2 \geq 2 m-3$ or, equivalently, $m \leq 1$, a contradiction.

(ii) $S$ is a finite simple group of Lie type in characteristic $p$, with $S \neq{ }^{2} F_{4}(2)^{\prime}$. As $|S|$ is always divisible by an odd prime $r \geq 5$, we have $n \geq 2 r \geq 10$ by (10-2). Let $\mathrm{St}_{S}$ denote the Steinberg character of $S$. We can check that $\operatorname{St}_{S}(1)=|S|_{p} \geq 4$. Since $\operatorname{St}_{S}(1) \in \operatorname{cd}(S), 2 \operatorname{St}_{S}(1)$ and $\operatorname{St}_{S}(1)^{2}$ are character degrees of $\widehat{S}_{n}$ by (10-1). As $2 \mathrm{St}_{S}(1)<\operatorname{St}_{S}(1)^{2}$, we have $\operatorname{St}(1)^{2}>d_{1}\left(\widehat{\mathrm{S}}_{n}\right)=n-1$. Since $n \geq 10$, Lemmas 2.1 and 2.3 yield that $\operatorname{St}_{S}(1)^{2}$ is a 2-power. Hence, $2 \mathrm{St}(1)$ is also a 2-power. By [TongViet 2012, Lemma 8], there exists a nontrivial character degree $x$ of $S$ such that $1<$ $x<\mathrm{St}_{S}(1)$. It follows that $2 x<2 \mathrm{St}_{S}(1)$ is also a character degree of $\widehat{\mathrm{S}}_{n}$. Therefore, $2 \mathrm{St}_{S}(1)>d_{1}\left(\widehat{\mathrm{S}}_{n}\right)=n-1$. Hence, $\widehat{\mathrm{S}}_{n}$ has two distinct nontrivial 2-power character degrees, neither of which is $n-1$. It follows that $n=2^{r}+2 \geq 10$, and furthermore

$$
2 \operatorname{St}_{S}(1)=2^{\lfloor(n-1) / 2\rfloor} \text { and } \operatorname{St}_{S}(1)^{2}=2^{2^{r-1}+r}
$$

by Lemma 2.3. We write $\operatorname{St}_{S}(1)=2^{N}$. Then $2^{r-1}+r=2 N$ and $2^{r-1}=N+1$ since $\lfloor(n-1) / 2\rfloor=2^{r-1}$. Solving these equations, we have $r=N-1$ and $2^{r-1}=r+2$. As $n \geq 10$, we deduce that $r \geq 3$. In this case, it is easy to check that the equation $2^{r-1}=r+2$ has no integer solution.

(iii) $S$ is a sporadic simple group or the Tits group. Since the arguments are fairly similar, we consider just the case $S=J_{3}$ as an example. Recall that $p(S)$ is the largest prime divisor of $|S|$. By (10-2), we have $n \geq 2 p(S)$. Since $n \geq 2 p(S) \geq 22$, we have $d_{2}\left(\widehat{\mathrm{S}}_{n}\right)=n(n-3) / 2 \geq p(S)(2 p(S)-3)$ by applying Lemma 3.5(3). For $i=1,2$, we have $2 d_{i}(S) \in \operatorname{cd}(G) \subseteq \operatorname{cd}\left(\hat{\mathrm{S}}_{n}\right)$ with $1<2 d_{1}(S)<2 d_{2}(S)$. For each possibility for $S$, we can check using [Conway et al. 1985] that $p(S)(2 p(S)-3)>$ $2 d_{2}(S)$; hence $d_{2}\left(\hat{\mathrm{S}}_{n}\right)>2 d_{2}(S)>2 d_{1}(S)$, which is a contradiction.

We are now ready to prove the main result, Theorem $\mathrm{C}$, which we restate below for the reader's convenience:

Theorem C. Let $n \geq 5$. Let $G$ be a finite group and $\widehat{\mathrm{S}}_{n}^{ \pm}$the double covers of $\mathrm{S}_{n}$. Then $\mathbb{C} G \cong \mathbb{C} \widehat{S}_{n}^{+}$(or equivalently $\mathbb{C} G \cong \mathbb{C} \widehat{S}_{n}^{-}$) if and only if $G \cong \widehat{\mathrm{S}}_{n}^{+}$or $G \cong \widehat{\mathrm{S}}_{n}^{-}$.

Proof. By the hypothesis that $\mathbb{C} G \cong \mathbb{C} \widehat{S}_{n}$, we have $|G|=2 n$ !, and, as $\widehat{S}_{n}$ has two linear characters, we also have $\left|G: G^{\prime}\right|=2$.

First we claim that $G^{\prime}=G^{\prime \prime}$. Assume not. Then $H:=G / G^{\prime}$ is a group whose commutator subgroup $H^{\prime}$ is nontrivial abelian of index 2. Now the induction of any nonprincipal (linear) character of $H^{\prime}$ to $H$ must be irreducible and 2-dimensional. This is not possible since $\widehat{\mathrm{S}}_{n}$ with $n \geq 5$ does not have any irreducible character of degree 2. Thus $G^{\prime}=G^{\prime \prime}$.

As $G^{\prime}=G^{\prime \prime}$ and $G^{\prime}$ is nontrivial, one can choose a normal subgroup $M$ of $G$ such that $M<G^{\prime}$ and

$$
G^{\prime} / M \cong S^{k}
$$


where $S$ is a nonabelian simple group and $S^{k}$ is a chief factor of $G$. Let

$$
C / M:=C_{G / M}\left(G^{\prime} / M\right) .
$$

(A) First we consider the case $C=M$. Then $G^{\prime} / M$ is the unique minimal normal subgroup of $G / M$. Therefore $G / M$ permutes the direct factors of $G^{\prime} / M$ (which is isomorphic to $S^{k}$ ). It then follows that $k \leq 2$ as $\left|G: G^{\prime}\right|=2$. Invoking Proposition 10.1, we deduce that $k=1$ and thus $G^{\prime} / M \cong S$. Therefore, $G^{\prime} / M$ is the socle of $G / M$. As $\operatorname{cd}^{*}(G / M) \subseteq \operatorname{cd}^{*}\left(\widehat{S}_{n}\right)$, Proposition 9.2 then implies that $G^{\prime} / M \cong \mathrm{A}_{n}$. Thus $G / M \cong \mathrm{S}_{n}$ and also $|M|=2$. In particular, $M$ is central in $G$ and therefore $M \subseteq Z(G) \cap G^{\prime}$. We conclude that $G$ is one of the two double covers of $\mathrm{S}_{n}$, as desired.

(B) It remains to consider the case $C>M$. Since $C / M \triangleleft G / M$ and $Z\left(G^{\prime} / M\right)=1$, it follows that $G^{\prime}$ is a proper subgroup of $G^{\prime} C$. As $\left|G: G^{\prime}\right|=2$, we then deduce that $G=G^{\prime} C$ and hence

$$
G / M=G^{\prime} / M \times C / M, \text { where } C / M \cong C_{2} .
$$

Applying Proposition 9.3, we obtain that $k=1$ and $S$ is isomorphic to $\mathrm{A}_{n}$. In other words, $G^{\prime} / M \cong \mathrm{A}_{n}$. So $G / M \cong \mathrm{A}_{n} \times C_{2}$. Comparing the orders, we get $|M|=2$ and so $M \subseteq \boldsymbol{Z}(G)$. As $M \leq G^{\prime}=G^{\prime \prime}$, it follows that $M \leq \boldsymbol{Z}\left(G^{\prime}\right) \cap G^{\prime \prime}$, which in turn implies that $G^{\prime}$ is the double cover of $\mathrm{A}_{n}$. We have proved that

$$
G^{\prime} \cong \widehat{\mathrm{A}}_{n}
$$

Moreover, as $C / M \cong C_{2}$ and $|M|=2$, we have

$$
C \cong C_{4} \text { or } C_{2} \times C_{2} .
$$

Now we claim that $G$ is an (internal) central product of $G^{\prime}$ and $C$ with amalgamated central subgroup $M$. To see this, let $x, y \in G^{\prime}$ and $c \in C$. Then the facts $C / M=C_{G / M}\left(G^{\prime} / M\right)$ and $M \leq Z(G)$ imply

$$
[x, y]^{c}=\left[x^{c}, y^{c}\right]=\left[x m_{1}, y m_{2}\right]=[x, y]
$$

for some $m_{1}, m_{2} \in M$. Therefore, $C$ centralizes $G^{\prime}=G^{\prime \prime}$ and the claim follows. This claim, together with (10-4) and (10-5), yield

$$
4 k\left(\widehat{\mathrm{A}}_{n}\right)=4 k\left(G^{\prime}\right)=k\left(G^{\prime} \times C\right) \leq k(M) k(G)=2 k(G),
$$

where the inequality comes from the well-known result that $k(X) \leq k(N) k(X / N)$ for $N$ a normal subgroup of $X$ (see [Nagao 1962] for instance). Since $\operatorname{cd}^{*}(G)=$ $\operatorname{cd}^{*}\left(\widehat{\mathrm{S}}_{n}\right)$, we have $k(G)=k\left(\widehat{\mathrm{S}}_{n}\right)$. It follows that $2 k\left(\widehat{\mathrm{A}}_{n}\right) \leq k\left(\widehat{\mathrm{S}}_{n}\right)$. This however contradicts Lemma 4.5, and the theorem is now completely proved. 


\section{Acknowledgements}

We close this article with many thanks to the referee for the careful reading of the manuscript and several helpful comments.

\section{References}

[Balog et al. 2001] A. Balog, C. Bessenrodt, J. B. Olsson, and K. Ono, "Prime power degree representations of the symmetric and alternating groups", J. London Math. Soc. (2) 64:2 (2001), 344-356. MR 2002g:20025 Zbl 1018.20008

[Bessenrodt and Olsson 2002] C. Bessenrodt and J. B. Olsson, "Prime power degree representations of the double covers of the symmetric and alternating groups", J. London Math. Soc. (2) 66:2 (2002), 313-324. MR 2003e:20012 Zbl 1018.20009

[Brauer 1963] R. Brauer, "Representations of finite groups", pp. 133-175 in Lectures on Modern Mathematics, I, edited by T. L. Saaty, Wiley, New York, 1963. MR 31 \#2314 Zbl 0124.26504

[Brunat 2010] O. Brunat, "Counting $p^{\prime}$-characters in finite reductive groups", J. London Math. Soc. (2) 81:3 (2010), 544-562. MR 2012a:20023 Zbl 1251.20017

[Conway et al. 1985] J. H. Conway, R. T. Curtis, S. P. Norton, R. A. Parker, and R. A. Wilson, Atlas of finite groups, Oxford University Press, 1985. MR 88g:20025 Zbl 0568.20001

[Frame et al. 1954] J. S. Frame, G. d. B. Robinson, and R. M. Thrall, "The hook graphs of the symmetric groups", Canadian J. Math. 6 (1954), 316-324. MR 15,931g Zbl 0055.25404

[Harborth and Kemnitz 1981] H. Harborth and A. Kemnitz, "Calculations for Bertrand's postulate", Math. Mag. 54:1 (1981), 33-34. MR 82c:10001 Zbl 0453.10006

[Hoffman and Humphreys 1992] P. N. Hoffman and J. F. Humphreys, Projective representations of the symmetric groups, Clarendon, New York, 1992. MR 94f:20027 Zbl 0777.20005

[Huppert 2000] B. Huppert, "Some simple groups which are determined by the set of their character degrees, I”, Illinois J. Math. 44:4 (2000), 828-842. MR 2001k:20009 Zbl 0972.20006

[Huppert and Lempken 2000] B. Huppert and W. Lempken, "Simple groups of order divisible by at most four primes”, Izv. Gomel. Gos. Univ. Im. F. Skoriny 2000:3(16) (2000), 64-75. Zbl 1159.20303

[Isaacs 1994] I. M. Isaacs, Character theory of finite groups, Dover, New York, 1994. Corrected reprint of the 1976 original. MR $1280461 \mathrm{Zbl} 0849.20004$

[Kleshchev and Tiep 2004] A. S. Kleshchev and P. H. Tiep, "On restrictions of modular spin representations of symmetric and alternating groups", Trans. Amer. Math. Soc. 356:5 (2004), 1971-1999. MR 2005a:20020 Zbl 1065.20013

[Kleshchev and Tiep 2012] A. S. Kleshchev and P. H. Tiep, "Small-dimensional projective representations of symmetric and alternating groups", Algebra Number Theory 6:8 (2012), 1773-1816. MR 3033527

[Lübeck 2001] F. Lübeck, "Smallest degrees of representations of exceptional groups of Lie type", Comm. Algebra 29:5 (2001), 2147-2169. MR 2002g:20029 Zbl 1004.20003

[Macdonald 1971] I. G. Macdonald, "On the degrees of the irreducible representations of symmetric groups”, Bull. London Math. Soc. 3 (1971), 189-192. MR 44 \#6865 Zbl 0219.20008

[Macdonald 1995] I. G. Macdonald, Symmetric functions and Hall polynomials, 2nd ed., Clarendon, New York, 1995. MR 96h:05207 Zbl 0824.05059

[McKay 1972] J. McKay, "Irreducible representations of odd degree", J. Algebra 20 (1972), 416-418. MR 44 \#4111 Zbl 0235.20009 
[Morris 1962] A. O. Morris, "The spin representation of the symmetric group", Proc. London Math. Soc. (3) 12 (1962), 55-76. MR 25 \#133 Zbl 0104.25202

[Nagao 1962] H. Nagao, "On a conjecture of Brauer for $p$-solvable groups", J. Math. Osaka City Univ. 13 (1962), 35-38. MR 27 \#2547 Zbl 0115.25501

[Navarro 2010] G. Navarro, "Problems in character theory", pp. 97-125 in Character theory of finite groups, Contemp. Math. 524, Amer. Math. Soc., Providence, RI, 2010. MR 2012a:20014 Zbl 1235.20009

[Nguyen 2010] H. N. Nguyen, "Low-dimensional complex characters of the symplectic and orthogonal groups”, Comm. Algebra 38:3 (2010), 1157-1197. MR 2011h:20025 Zbl 1237.20012

[Nguyen 2013] H. N. Nguyen, "Quasisimple classical groups and their complex group algebras", Israel J. Math. 195:2 (2013), 973-998. MR 3096579 Zbl 1294.20004

[Nguyen and Tong-Viet 2014] H. N. Nguyen and H. P. Tong-Viet, "Characterizing finite quasisimple groups by their complex group algebras”, Algebr. Represent. Theory 17:1 (2014), 305-320. MR 3160726 Zbl 1303.20001

[Olsson 1993] J. B. Olsson, Combinatorics and representations of finite groups, Fachbereich Mathematik der Universität Essen, Heft 20, 1993. MR 95b:20020 Zbl 0796.05095

[Rasala 1977] R. Rasala, "On the minimal degrees of characters of $S_{n}$ ", J. Algebra 45:1 (1977), 132-181. MR 55 \#477 Zbl 0348.20009

[Schur 1911] I. Schur, "Über die Darstellung der symmetrischen und der alternierenden Gruppe durch gebrochene lineare Substitutionen”, J. Reine Angew. Math. 139 (1911), 155-250.

[Tiep and Zalesskii 1996] P. H. Tiep and A. E. Zalesskii, "Minimal characters of the finite classical groups”, Comm. Algebra 24:6 (1996), 2093-2167. MR 97f:20018 Zbl 0901.20031

[Tong-Viet 2011] H. P. Tong-Viet, "Symmetric groups are determined by their character degrees", J. Algebra 334 (2011), 275-284. MR 2012c:20020 Zbl 1246.20007

[Tong-Viet 2012] H. P. Tong-Viet, "Alternating and sporadic simple groups are determined by their character degrees”, Algebr. Represent. Theory 15:2 (2012), 379-389. MR 2892513 Zbl 1252.20005

[Wagner 1977] A. Wagner, "An observation on the degrees of projective representations of the symmetric and alternating group over an arbitrary field", Arch. Math. 29:6 (1977), 583-589. MR 57 \#444 Zbl 0383.20009

[Wales 1979] D. B. Wales, "Some projective representations of $S_{n}$ ", J. Algebra 61:1 (1979), 37-57. MR 81f:20015 Zbl 0433.20010

Communicated by David Benson

Received 2014-05-19 Revised 2015-01-13 Accepted 2015-02-23

bessen@math.uni-hannover.de Institut für Algebra, Zahlentheorie und Diskrete Mathematik, Fakultät für Mathematik und Physik, Leibniz Universität Hannover, Welfengarten 1, D-30167 Hannover, Germany

hungnguyen@uakron.edu

Department of Mathematics, The University of Akron, Akron, $\mathrm{OH} 44325$, United States

olsson@math.ku.dk

Department of Mathematical Sciences,

University of Copenhagen, DK-2100 Copenhagen $\varnothing$, Denmark

Hung.Tong-Viet@up.ac.za Department of Mathematics and Applied Mathematics,

University of Pretoria, Private Bag X20,

Hatfield, Pretoria 0002, South Africa 


\section{Algebra \& Number Theory}

msp.org/ant

\section{EDITORS}

MANAGING EDITOR

Bjorn Poonen

Massachusetts Institute of Technology

Cambridge, USA

\author{
EDITORIAL BOARD CHAIR \\ David Eisenbud \\ University of California \\ Berkeley, USA
}

\section{BOARD OF EDITORS}

Georgia Benkart

Dave Benson

Richard E. Borcherds

John H. Coates

J-L. Colliot-Thélène

Brian D. Conrad

Hélène Esnault

Hubert Flenner

Edward Frenkel

Andrew Granville

Joseph Gubeladze

Roger Heath-Brown

Craig Huneke

János Kollár

Yuri Manin

Barry Mazur

Philippe Michel
University of Wisconsin, Madison, USA

University of Aberdeen, Scotland

University of California, Berkeley, USA

University of Cambridge, UK

CNRS, Université Paris-Sud, France

University of Michigan, USA

Freie Universität Berlin, Germany

Ruhr-Universität, Germany

University of California, Berkeley, USA

Université de Montréal, Canada

San Francisco State University, USA

Oxford University, UK

University of Virginia, USA

Princeton University, USA

Northwestern University, USA

Harvard University, USA

École Polytechnique Fédérale de Lausanne
Susan Montgomery

Shigefumi Mori

Raman Parimala

Jonathan Pila

Anand Pillay

Victor Reiner

Peter Sarnak

Joseph H. Silverman

Michael Singer

Vasudevan Srinivas

J. Toby Stafford

Ravi Vakil

Michel van den Bergh

Marie-France Vignéras

Kei-Ichi Watanabe

Efim Zelmanov

Shou-Wu Zhang
University of Southern California, USA

RIMS, Kyoto University, Japan

Emory University, USA

University of Oxford, UK

University of Notre Dame, USA

University of Minnesota, USA

Princeton University, USA

Brown University, USA

North Carolina State University, USA

Tata Inst. of Fund. Research, India

University of Michigan, USA

Stanford University, USA

Hasselt University, Belgium

Université Paris VII, France

Nihon University, Japan

University of California, San Diego, USA

Princeton University, USA

\section{PRODUCTION}

production@msp.org

Silvio Levy, Scientific Editor

See inside back cover or msp.org/ant for submission instructions.

The subscription price for 2015 is US $\$ 255 /$ year for the electronic version, and $\$ 440 /$ year ( $+\$ 55$, if shipping outside the US) for print and electronic. Subscriptions, requests for back issues and changes of subscribers address should be sent to MSP.

Algebra \& Number Theory (ISSN 1944-7833 electronic, 1937-0652 printed) at Mathematical Sciences Publishers, 798 Evans Hall \#3840, c/o University of California, Berkeley, CA 94720-3840 is published continuously online. Periodical rate postage paid at Berkeley, CA 94704, and additional mailing offices.

ANT peer review and production are managed by EditFLOW ${ }^{\circledR}$ from MSP.

\section{PUBLISHED BY}

- mathematical sciences publishers

nonprofit scientific publishing

http://msp.org/

(C) 2015 Mathematical Sciences Publishers 


\section{Algebra \& Number Theory}

Volume $9 \quad$ No. $3 \quad 2015$

Hurwitz monodromy and full number fields

DAVID P. ROBERTS and AKSHAY VENKATESH

The characteristic polynomial of the Adams operators on graded connected Hopf algebras

Marcelo Aguiar and Aaron Lauve

Secant spaces and syzygies of special line bundles on curves

MARIAN APRODU and EDOARDO SERNESI

Complex group algebras of the double covers of the symmetric and alternating groups

601

Christine Bessenrodt, Hung Ngoc Nguyen, Jørn B. Olsson and Hung P. TONG-VIET

Fano schemes of determinants and permanents

Melody Chan and NATHan Ilten

Triple intersection formulas for isotropic Grassmannians

VIJAY RAVIKUMAR

On the basepoint-free theorem for log canonical threefolds over the algebraic closure of a finite field

Diletta Martinelli, Yusuke NaKamura and Jakub Witaszek

The torsion group of endotrivial modules

JON F. CARLSON and JACQUES THÉVENAZ 